\title{
RECURSIVELY ENUMERABLE SETS AND DEGREES
}

\author{
BY ROBERT I. SOARE ${ }^{1}$ \\ TABLE OF CONTENTS \\ Introduction
}

Chapter I. The relation of the structure of an r.e. set to its degree.

1. Post's program and simple sets.

2. Dominating functions and quotient lattices.

3. Maximal sets and high degrees.

4. Low degrees, atomless sets, and invariant degree classes.

5. Incompleteness and completeness for noninvariant properties.

Chapter II. The structure, automorphisms, and elementary theory of the r.e. sets.

6. Basic facts and splitting theorems.

7. Hh-simple sets.

8. Major subsets and $r$-maximal sets.

9. Automorphisms of $\mathcal{E}$.

10. The elementary theory of $\mathcal{E}$.

Chapter III. The structure of the r.e. degrees.

11. Basic facts.

12. The finite injury priority method.

13. The infinite injury priority method.

14. The minimal pair method and lattice embeddings in $\mathbf{R}$.

15. Cupping and splitting r.e. degrees.

16. Automorphisms and decidability of $\mathbf{R}$.

Introduction. G. E. Sacks has remarked that recursion theory is the heart of logic, and recursively enumerable sets form the soul of recursion theory. Although some might challenge these claims, it is clear that recursively enumerable sets have played an important role in logic beginning with the first undecidability results of Gödel [Gö1], Church [Ch] and Rosser [Rs]. Furthermore, the notion of a recursively enumerable set rather than that of a recursive (i.e, computable) function has proved to be the fundamental concept in attempts to generalize classical recursion theory to more general settings, such as admissible ordinals [Sh4], [Le6], or higher types [Sa9].

A subset $\boldsymbol{A}$ of $\boldsymbol{\omega}$ (the set of nonnegative integers) is recursive (also called decidable or computable) if there is an algorithm for determining whether a

This is an expanded version of an Invited Address given at the 84th Annual Meeting of the American Mathematical Society in Atlanta, Georgia, on January 5, 1978; received by the editors November 10, 1977 and, in revised form, December 5, 1977.

AMS (MOS) subject classifications (1970). Primary 02F25, 02F30; Secondary 02F47, 02G05, 02G10, 06A20, 02F50.

'The author was partially supported by NSF grant MCS 76-07033 A01.

- American Mathematical Society 1978 
number is in $A$, and $A$ is recursively enumerable (r.e.) if there is an algorithm for enumerating the members of $A$.

Recursively enumerable sets are important because they are the next most effective type of set beyond recursive sets and they occur naturally in many branches of mathematics. This widespread occurrence of r.e. sets together with the existence of nonrecursive r.e. sets has enabled them to play a key role in famous results such as Gödel's incompleteness theorem, Hilbert's tenth problem on Diophantine equations, and the unsolvability of the word problem for finitely presented groups.

A set $A \subseteq \omega$ is Diophantine if there is a polynomial $p\left(x, y_{1}, y_{2}, \ldots, y_{m}\right)$ with integral coefficients (positive or negative) such that

$$
x \in A \Leftrightarrow\left(\exists y_{1}\right)\left(\exists y_{2}\right) \cdots\left(\exists y_{m}\right)\left[p\left(x, y_{1}, \ldots, y_{m}\right)=0\right] \text {. }
$$

Clearly, every Diophantine set is r.e. The remarkable result of Matiyasevič [Mt1], [Mt2] that every r.e. set is Diophantine demonstrates the abundance of r.e. sets arising naturally in number theory, and simultaneously yields a negative answer to Hilbert's tenth problem, namely there is no algorithm to determine for a given polynomial Diophantine equation with integral coefficients whether it has a solution in the integers.

For sets $A, B \subseteq \omega, A$ is recursive in (also called Turing reducible to) a set $B$, written $A<T B$, if there is an algorithm for deciding whether $x \in A$ provided we are given answers to all questions of the form "Is $y \in B$ ?". Sets $A$ and $B$ have the same degree $\left(A \equiv_{T} B\right)$ if $A<_{T} B$ and $B<_{T} A$. The equivalence class of $A$ under the equivalence relation $\equiv_{T}$ is the degree of $A$, written $\operatorname{dg}(A)$, and $\operatorname{dg}(A)<\operatorname{dg}(B)$ iff $A<T B$. A degree is r.e. if it contains an r.e. set.

Let $S$ be a set and $D$ a collection of words on the elements of $S$ and their inverses. The group $G$ presented by $\langle S ; D\rangle$ is the quotient group of the free group on $S$ by the normal closure of the words in $D$, and $G$ is finitely presented if both $S$ and $D$ are finite. The word problem for $G$ is the problem of deciding given an arbitrary word $w$ of $G$ whether $w=1$ in $G$. Clearly the set $A_{G}$ of such words $w$ is r.e. (under some effective coding of words by integers) but Boone [Bo1], [Bo2] and Novikov [No] proved that $A_{G}$ is not always recursive, and hence the word problem for finitely presented groups is unsolvable. Indeed Boone [B03] showed that for any r.e. set $B$ there is a finitely presented group whose word problem has the same degree as $B$. Thus, all r.e. degrees (if not all r.e. sets) arise as word problems in group theory.

Instead of considering these applications of r.e. sets for which there are already excellent expositions [Da3] and [Mr], we concentrate here on the pure theory of r.e. sets as initiated by Post in his epochal 1944 address before this Society [Po]. Post stripped away the formalism associated with the development of recursive functions in the 1930's and revealed in a clear informal style the essential properties of r.e. sets and their application to Gödel's incompleteness theorem. Post went on to attempt to classify the r.e. sets and their degrees. He raised the question which became known as Post's problem: does there exist more than one nonrecursive r.e. degree? The existence of infinitely many such degrees was proved much later and implies for example that there are infinitely many genuinely different unsolvable word problems for finitely presented groups rather than just one. 
The purpose of this paper is to give a survey of the main ideas and results on r.e. sets from Post's time up to the present state of the subject and latest research. Sketches of proofs will be given to illustrate important methods, but technical details will be kept to a minimum. The paper is intended for one who is not an expert in logic or recursive function theory.

Most of the material will be written in expanded form with complete proofs in a forthcoming manuscript [So5]. Excellent introductions to the subject are Rogers [Rg2] and Shoenfield [Sf5].

We begin in Chapter I with a study of Post's program and the results it stimulated which concern the relationship between the structure of an r.e. set and its degree. In Chapter II we examine the lattice of r.e. sets, its structure, its automorphisms, and the question of decidability of its elementary theory. In Chapter III we consider the same question for the r.e. degrees $\mathbf{R}$.

\section{CHAPTER I. THE RELATION OF THE STRUCTURE OF AN R.E. SET TO ITS DEGREE}

1. Post's program and simple sets. (All sets will be subsets of $\omega$ and all functions will be on $\omega$.) A partial recursive (p.r.) function (from $\omega$ to $\omega$ ) is a partial function whose graph is r.e. A recursive function is a p.r. function which is total, i.e., domain $f=\omega$. Since we can effectively list all algorithms we can effectively list all r.e. sets $\left\{W_{e}\right\}_{e \in \omega}$ and all p.r. functions $\left\{\varphi_{e}\right\}_{e \in \omega}$ but not all total recursive functions since we cannot decide given $\varphi_{e}$ and $x$ whether $\varphi_{e}(x)$ is defined. A simultaneous enumeration of the r.e. sets is a recursive function $f$ with range $\left\{\langle x, e\rangle: x \in W_{e}\right\}$ and $W_{e, s}=\{x:(\exists t<$ $s)[f(t)=\langle x, e\rangle]\}$. (Let $\langle x, y\rangle$ denote the image of the ordered pair $(x, y)$ under some effective 1: 1 map from $\omega \times \omega$ onto $\omega$.)

Notice that the r.e. sets form a distributive lattice $\mathcal{E}$ since if $A$ and $B$ are r.e. then $A \cup B$ and $A \cap B$ are r.e. It is easy to see that a set $A$ is recursive (has a recursive characteristic function $\chi_{1}$ ) iff both $A$ and $\bar{A}$ are r.e. (To compute $\chi_{1}(n)$ simultaneously enumerate $A$ and $\bar{A}$ until $n$ appears in one of them.) Thus the recursive sets form a Boolean algebra $R$ consisting precisely of the complemented members of $\mathcal{E}$. Now by simultaneously enumerating all r.e. sets one can construct the r.e. set $K=\left\{e: e \in W_{e}\right\}$, which must be nonrecursive since $\bar{K} \neq W_{e}$ for any $e$.

Notice that $K$ is effectively noncomplemented (and therefore effectively nonrecursive) in the sense that there is a recursive function $f$ (the identity function in the case of $K)$ such that $W_{e} \subset \bar{K}$ implies $f(e) \in \bar{K}-W_{e}$. Such r.e. sets were called creative by Post [Po] because their existence together with the representation of all r.e. sets in such a fragment of mathematics as elementary number theory implies the impossibility of mechanically listing all statements true in such a fragment. Post remarks: "The conclusion is unescapable that even for such a fixed, well defined body of mathematical propositions, mathematical thinking is, and must remain, essentially creative."

Myhill later proved [My1] that any two creative sets $A$ and $B$ are recursively isomorphic, namely there is a recursive permutation $f$ of $\omega$ such that $f(A)=B$. Hence, there is really only one creative set up to recursive isomorphism. An r.e. set $A$ is complete (Turing complete) if $W_{e} \leqslant_{T} A$ for 
every $e$. The set $K$ (and hence all creative sets) are complete. Post's problem was to construct a nonrecursive r.e. set $A$ which is incomplete, namely $\varnothing<_{T} A<_{T} K$. Post's program for constructing $A$ was to find an easily definable property of $\bar{A}$ (compatible with $A$ nonrecursive) which would guarantee incompleteness.

Now if $C$ is creative (via say $f$ ) then $\bar{C}$ must contain an infinite r.e. set $\left\{a_{n}\right\}_{n \in \omega^{*}}$ (Let $a_{0}=f\left(x_{0}\right)$, where $W_{x_{0}}=\varnothing$, and $a_{k+1}=f\left(x_{k}\right)$ where $W_{x_{k}}=$ $\left\{a_{0}, a_{1}, \ldots, a_{k}\right\}$.) Post's idea for constructing $A$ incomplete was to make $\bar{A}$ sufficiently "thin" with respect to containment of r.e. sets so that $\bar{K}$ could not be Turing reduced to $\bar{A}$. He defined an r.e. set $S$ to be simple if $\bar{S}$ is infinite but $\bar{S}$ contains no infinite r.e. sets. As a special case of Turing reducibility Post defined $A$ to be $m$-reducible to $B(A \leqslant m B)$ if there is a recursive function $f$ such that $f(A) \subseteq B$ and $f(\bar{A}) \subseteq \bar{B}$. Post constructed a simple set and proved that any simple set $A$ is $m$-incomplete, namely $K \sharp_{m} A$. However, Post realized that simple sets could be (Turing) complete and thus he defined r.e. sets with even thinner complements called hypersimple ( $h$-simple) and hyperhypersimple ( $h h$-simple).

If $x_{1}<x_{2}<\cdots<x_{k}$ and $y=2^{x_{1}}+\cdots+2^{x_{k}}$ let $D_{y}$ denote the finite set $\left\{x_{1}, x_{2}, \ldots, x_{k}\right\}$. A sequence $\left\{F_{n}\right\}_{n \in \omega}$ of finite sets is a strong (weak) array if there is a recursive function $f$ such that $F_{n}=D_{f(n)}\left(F_{n}=W_{f(n)}\right)$ for all $n$. An array is disjoint if its members are pairwise disjoint. A coinfinite r.e. set $A$ is $h$-simple ( $h h$-simple) if there is no strong (weak) disjoint array $\left\{F_{n}\right\}_{n \in \omega}$ such that $F_{n} \cap \bar{A} \neq \varnothing$ for all $n$.

(The intention is that instead of specifying an infinite r.e. set $\left\{a_{n}\right\}_{n \in \omega} \subseteq \bar{A}$, we specify an array $\left\{F_{n}\right\}_{n \in \omega}$ such that each $F_{n}$ contains some $a_{n} \in \bar{A}$ but we cannot tell which $x \in F_{n}$ has this property. In a strong array we can explicitly compute $\max \left(F_{n}\right)$ and all its members, whereas in a weak array we can merely enumerate $F_{n}$.)

Post constructed an $h$-simple set $H$, proved that $A h$-simple implies $K$ $\$_{t} \boldsymbol{A}$ for a certain intermediate "tt-reducibility," but he was unable to construct an $h h$-simple set or to prove that this property guaranteed (Turing) incompleteness.

2. Dominating functions and quotient lattices. More than ten years passed with little progress on r.e. sets until two previous mathematical ideas were applied with ultimately fertile consequences for $\mathcal{E}$, namely dominating functions and maximal elements in quotient lattices of $\mathcal{E}$. The idea of dominating functions arose in the classification of $h$-simple sets. A function $f$ dominates a function $g$ if $f(n) \geqslant g(n)$ for all but finitely many $n$ (a.e. $n$ ). If $A=a_{0}<a_{1}<\ldots$ is an infinite set the principal function of $A$ is $p_{A}$ where $p_{A}(n)=a_{n}$. A function $f$ dominates an infinite set $A$ if it dominates $p_{A}$. Let $[m, n]$ be the closed interval $\{m, m+1, \ldots, n\},(m, n]=\{m+1, \ldots, n\}$ and so on. Let $A[n]=A \cap[0, n]$, and $|A|$ denote the cardinality of $A$.

TheOREM 2.1 (KuZNeCOV, MEDVEDEV, USPENSKIII [Md]). An r.e. set $A$ is $h$-simple iff no recursive function dominates $\bar{A}$.

Proof. $\Leftarrow$. Let $\left\{D_{g(x)}\right\}_{x \in \omega}$ be a disjoint strong array such that $D_{g(x)} \cap \bar{A}$ $\neq \varnothing$ for all $x$. Let $f(x)=\max \cup\left\{D_{g(y)}: y<x\right\}$. 
$\Rightarrow$ : By adjusting $f$ on finitely many numbers we may assume $f(n) \geqslant p_{\bar{A}}(n)$ for all $n$. Set $D_{g(0)}=[0, f(0)]$. Given $g(0), \ldots, g(n)$, let $k=\max \cup\left\{\mathrm{D}_{g(i)}\right.$ : $\mathrm{i} \leq \mathrm{n}\}$, and set $D_{g(n+1)}=[k+1, f(k+2)]$. Now $|\bar{A}[f(k+2)]| \geqslant k+2$, but $|\bar{A}[k]| \leqslant k+1$, so $D_{g(n+1)} \cap \bar{A} \neq \varnothing$.

Using this characterization Dekker [Dk2] proved that for every nonrecursive r.e. set $A$ there is an $h$-simple set $B \equiv_{T} A$. (Let $\left\{a_{s}\right\}_{s \in \omega}$ be a recursive enumeration of $A$ and let $B=\left\{s:(\exists t>s)\left[a_{t}<a_{s}\right]\right\}$, the deficiency set of $A$ for this enumeration. Clearly $B \equiv_{T} A, B$ is r.e. and any recursive function dominating $\bar{B}$ would imply $\boldsymbol{A}$ recursive.) Let $\mathbf{R}^{+}$denote the nonrecursive r.e. degrees, $\mathbf{R}-\{\boldsymbol{0}\}$, where $\mathbf{0}$ is the degree consisting of the recursive sets. If $\mathcal{C} \subseteq \mathcal{E}$, let $\operatorname{dg}(\mathcal{C})=\{\operatorname{dg}(W): W \in \mathcal{C}\}$

THEOREM 2.2 (DEKKER). Let $\delta$ be the class of simple sets. Then $\operatorname{dg}(\delta)=$ $\mathbf{R}^{+}$.

Next Myhill [My2] suggested studying quotient lattices of $\mathcal{E}$ and maximal sets. Let $A={ }^{*} B$ denote that the symmetric difference of $A$ and $B$ is finite and $A \subseteq \subseteq^{*} B$ denote that $A \cap \bar{B}=* \varnothing$. Let $\mathcal{E}^{*}$ denote the quotient lattice of $\mathcal{E}$ modulo the ideal $\mathscr{F}$ of finite sets, and for $A \in \mathcal{E}$ let $A^{*}$ be the equivalence class of $A$ in $\mathcal{E}^{*}$. Thus, $A^{*}=B^{*}$ iff $A={ }^{*} B$. For any lattice $\mathcal{L} \subseteq \mathcal{E}$ closed under finite differences let $\mathfrak{L}^{*}=\left\{A^{*}: A \in \mathcal{L}\right\}$. It is very easy to see [La8, Lemma 14] or [So3, Theorem 1.3] that if $\varrho_{1}$ and $\varrho_{2}$ are any two such lattices then $\mathscr{L}_{1} \cong \mathscr{L}_{2}$ iff $\mathscr{L}_{1}^{*} \cong \mathscr{L}_{2}^{*}$ so it suffices to classify $\mathcal{L}^{*}$ in place of $\mathcal{L}$.

Myhill defined a coinfinite r.e. set $M$ to be maximal just in case $M^{*}$ is a coatom of $\mathcal{E}^{*}$, i.e. there is no $A \in \mathcal{E}$ such that $M \subset_{\infty} A \subset_{\infty} \omega$. (Let $X \subset \subset_{\infty} Y$ denote that $X \subseteq Y$ and $|Y-X|=\infty$.) Clearly every maximal set is $h h$-simple and indeed maximal sets have the thinnest possible complements in the sense of Post. Myhill asked whether maximal sets exist and noted that this is equivalent to $\mathcal{E}^{*}$ not being densely ordered. (Myhill also noted that the existence of a creative set $C$ implies that $\mathcal{E}^{*}$ is not Brouwerian since $C^{*}$ has no pseudo-complement [Bi2, p. 45], and that an r.e. set $A$ is simple iff $A^{*}$ is pseudo-complemented with trivial pseudo-complement.)

3. Maximal sets and high degrees. Suddenly Friedberg [Fr2] and independently Muchnik [Mu] solved Post's problem by an entirely different method now known as the finite injury priority method which we discuss in $\$ 12$. Indeed the method easily yielded infinitely many different r.e. degrees [Fr1].

THEOREM 3.1 (FRIEDBERG-MUCHNIK). There exist r.e. sets $A$ and $B$ such that $A \$ T B$ and $B \$_{T} A$. (Hence $\phi<_{T} A<_{T} K$ and $\phi<_{T} B<_{T} K$.)

Then Friedberg used a different priority method [Fr4] to construct a maximal set, and Yates later proved [Y2] that maximal sets can be complete, thereby refuting Post's idea that thinness of $\bar{A}$ implies $A$ incomplete. Still Sacks asked [Sa7, p. $172 \mathrm{Q}$ ] ] for some simple property of complements of r.e. sets (in the style of Post) which implies incompleteness. Soare [So7] gave a negative answer for all those properties which are lattice invariant (invariant under Aut $\mathcal{E}$, the group of automorphisms of $\mathcal{E}$ ).

THEOREM 3.2 (SOARE). For any nonrecursive r.e. set $A$ there exists $\Phi \in$ Aut $\mathcal{E}$ such that $\Phi(A) \equiv_{T} K$. 
We now sketch the Friedberg maximal set construction (as simplified by Yates [Y2]) which has been one of the major tools in the investigation of $\mathcal{E}$.

THEOREM 3.3 (FrIEDBERG). There exists a maximal set $M$.

SKETCH OF PROOF. We enumerate $M$ by stages and let $M_{s}$ denote the elements enumerated in $M$ by the end of stage $s$. It suffices to make $\bar{M}$ infinite and to meet for each $e$ the requirement

$$
P_{e}: W_{e} \cap \bar{M} \text { infinite } \Rightarrow \bar{M} \subseteq{ }^{*} W_{e}
$$

Let $\left\{\Lambda_{e}\right\}_{e \in \omega}$ be a sequence of "movable markers" which come to rest on the elements of $\bar{M}$. Let $m_{e}^{s}$ denote the position of $\Lambda_{e}$ at the end of stage $s$ and assume that $\bar{M}_{s}=m_{0}^{s}<m_{1}^{s}<\ldots$ Let $M_{0}=\varnothing$. To meet requirement $P_{0}$ choose at stage $s+1$ the least $i$ such that $m_{i}^{s} \notin W_{0, s}$ and $m_{j}^{s} \in W_{0, s}$ for some $j>i$. Move $\Lambda_{i}$ to $m_{j}^{s}, \Lambda_{i+1}$ to $m_{j+1}^{s}$, and so forth. Enumerate in $M_{s+1}$ all elements not under markers. Clearly, a similar strategy for $P_{1}$ can be played within this strategy for $P_{0}$, and so forth for all $P_{n}$.

As a bookkeeping device which gives priority first to $P_{0}$, then to $P_{1}$, and so on, define the e-state of $x$ at stage $s, E(e, x, s)=\Sigma\left\{2^{e-i}: i<e\right.$ and $x \in$ $\left.W_{i, s}\right\}$. Marker $\Lambda_{e}$ moves at stage $s+1$ to maximize its $e$-state. After all $\Lambda_{i}$, $i<e$, have settled $\Lambda_{e}$ moves at most $2^{e+1}$ times. Thus, all markers come to rest, $\bar{M}$ is infinite and satisfies $P_{e}$ for all $e$.

An infinite set $A$ dominates a partial function $\psi$ if $p_{A}(n) \geqslant \psi(n)$ for a.e. $n$ such that $\psi(n)$ is defined. If $\boldsymbol{A}$ dominates every partial recursive function then $A$ is necessarily complete because $A$ dominates $\psi_{K}(x)=(\mu s)\left[x \in K_{s}\right]$ if $s$ exists and $\psi_{K}(x)$ is undefined otherwise. (Let $(\mu y) P(y)$ denote the least $y$ such that $P(y)$ if such $y$ exists.) Reflecting upon this and Theorem 2.1, Tennenbaum suggested [Te1] just the opposite of Post's approach namely that perhaps every maximal set $M$ is complete because $\bar{M}$ dominates all partial recursive functions. Sacks refuted this suggestion by constructing an incomplete maximal set [Sa4], and he asked for a characterization of $\operatorname{dg}(\mathscr{T})$ where $\mathfrak{T}$ is the class of maximal sets. Tennenbaum [Te2] and independently Martin [Ma1] and [Ma3] noticed that Tennenbaum's suggestion is correct if partial recursive functions are replaced by total recursive functions. A function $f$ (set $A)$ is dominant if $f\left(p_{A}\right)$ dominates all total recursive functions.

Proposition 3.4 (Martin-Tennenbaum). If $M$ is maximal then $\bar{M}$ is dominant.

Proof. Suppose to the contrary that $\bar{M}$ fails to dominate some recursive function $f$. By Theorem $2.1 f$ does not dominate $\bar{M}$. Hence, there are infinitely many "crossover points" $x$ such that $f(x)<p_{\bar{M}}(x)$ but $p_{\bar{M}}(x+1)<f(x+$ $1)$, and hence $\left|\bar{M} \cap I_{x}\right| \geqslant 2$ where $I_{x}=(f(x), f(x+1)]$. Now construct $A \in \mathcal{E}$, such that $M \subset_{\infty} A \subset_{\infty} \omega$ by enumerating $x$ in $A$ at stage $s+1$ if $x \in M_{s}$ or $x=(\mu y)\left[y \in I_{z}-M_{s}\right]$ for some $z$.

Martin defined a coinfinite r.e. set $A$ to be dense simple if $\bar{A}$ is dominant and showed [Ma1] by a more subtle argument that not only maximal sets but all $h h$-simple sets are dense simple. Martin then used this notion of dominance to obtain his remarkable characterization of the degrees of maximal sets in terms of the jump operator. For any set $A$ define the jump of 
$A, A^{\prime}=\left\{n: n \in W_{n}^{A}\right\}$, where $W_{n}^{A}$ is the $n$th set which is r.e. in $A$ (r.e. given $A$ as an "oracle"). It is easily seen that $A^{\prime}$ is r.e. in $A$ but not recursive in $A$, and that the jump operator is well-defined on degrees, where $\operatorname{dg}(A)^{\prime}=\operatorname{dg}\left(A^{\prime}\right)$. (Note that $0^{\prime}=\operatorname{dg}(K)$ since $K$ is recursively isomorphic to $\varnothing^{\prime}$.) Let $\mathrm{a}^{(n+1)}=$ $\left(\mathfrak{a}^{(n)}\right)^{\prime}$. For each $n \geqslant 0$ define the subclasses of the r.e. degrees $\mathbf{R}$,

$$
\begin{aligned}
\mathbf{H}_{n} & =\left\{\mathrm{d}: \mathrm{d} \in \mathbf{R} \text { and } \mathrm{d}^{(n)}=\mathbf{0}^{(n+1)}\right\}, \text { and } \\
\mathbf{L}_{n} & =\left\{\mathrm{d}: \mathrm{d} \in \mathbf{R} \text { and } \mathrm{d}^{(n)}=\mathbf{0}^{(n)}\right\},
\end{aligned}
$$

where $d^{(0)}=\mathbf{d}$, and $\overline{\mathbf{L}}_{n}=\mathbf{R}-\mathbf{L}_{n}$. (Note that $\mathbf{R}^{+}=\overline{\mathbf{L}}_{0}$.) The degrees in $\mathbf{H}_{1}$ $\left(\mathbf{L}_{1}\right)$ are called high (low) since they have the highest (lowest) possible jump. An r.e. set $A$ is high (low) if $\operatorname{dg}(A) \in \mathbf{H}_{1}\left(L_{1}\right)$. It is known (see 813) that $\mathbf{H}_{n} \subsetneq \mathbf{H}_{n+1}$ and $\mathbf{L}_{n} \subsetneq \mathbf{L}_{n+1}$. Lachlan [La2], Martin [Ma4] and Sacks [Sa8] showed that $\left.\mathbf{R} \neq \cup^{\neq}\left(\mathbf{L}_{n} \cup \mathbf{H}_{n}\right): n \in \omega\right\}$.

The crucial breakthrough in the classification of $\operatorname{dg}(\mathfrak{T})$ was Martin's observation.

THEOREM 3.5 (MARTIN). An r.e. degree $a$ is in $\mathbf{H}_{1}$ iff there is a function $f$ of degree $<$ a which is dominant.

(The proof uses the fact that $\left\{e: \varphi_{e}\right.$ total $\}$ has degree $0^{n}$ and the Limit Lemma [Sf5, p. 29] which asserts that a function $g$ is recursive in $A^{\prime}$ iff $g(x)=\lim _{s} \hat{g}(x, s)$ for some function $\hat{g}$ recursive in $A$.)

THEOREM 3.6 (MARTIN). Let Th be the class of maximal sets. Then $\operatorname{dg}(\Re)$ $=\mathbf{H}_{\mathbf{1}}$.

SKETCH OF PROOF. If a contains a maximal set then $a \in \mathbf{H}_{1}$ by Proposition 3.4 and Theorem 3.5. Conversely, given a high degree a one can easily find [Ro5] an r.e. set $A \in \mathrm{a}$ and a recursive enumeration of $A$ whose computation function $C_{A}(x)$ is dominant where we define $C_{A}(x)=(\mu s)\left[A_{s}[x]=A[x]\right]$. Now combine the maximal set construction of Theorem 3.3 with a method of Yates [Y2] where an element $x$ is "permitted" to be enumerated in $M$ at stage $s+1$ only if $A_{s+1}[x] \neq A_{s}[x]$, whence $C_{M}(x)<C_{A}(x)$ and hence $M<T A$.

The key point is that the dominance of $C_{A}$ allows each requirement $P_{e}$ of (3.1) to be satisfied. Suppose for example that $P_{0}$ is not satisfied because infinitely many elements $x \in \bar{M}_{s} \cap \bar{W}_{0}$ are never permitted to enter $M$. Then $C_{A}$ fails to dominate the recursive function $f$, where $f(x)$ is the least $s$ such that: $x \in M_{s}$; or $x \in W_{0, s}$; or $x$ "wants" to enter $M$ at stage $s$ for the sake of $P_{0}$.

Finally, insure $A<r M$ as follows. When $n \in A_{s+1}-A_{s}$, enumerate in $M_{s+1}$ whichever of $m_{n}^{s}$ and $m_{n+1}^{s}$ has lower $n$-state at stage $s+1$. Thus if $m_{n}^{s}=m_{n}\left(=\lim _{s} m_{n}^{s}\right)$ then $A[n]=A_{s}[n]$.

Other classes $e$ such that $\operatorname{dg}(\mathcal{C})=\mathbf{H}_{1}$ are the dense simple sets (and therefore the $h h$-simple sets), and the coinfinite sets with no dense simple superset [Ma3].

4. Low degrees, atomless sets, and invariant degree classes. $A$ class $C$ of r.e. degrees is invariant if $\mathbf{C}=\mathrm{dg}(\mathcal{C})$ for some class $\mathcal{C} \subseteq \mathcal{E}$ invariant under Aut $\mathcal{E}$. Martin asked which other degree classes are invariant besides $H_{1}$ (and the trivially invariant classes $R, \mathbf{L}_{0}$ and $\mathbf{R}^{+}=\overline{\mathbf{L}}_{0}$ ). In particular, he asked 
[Ma3] for a classification of the degrees of atomless sets, coinfinite r.e. sets with no maximal superset. Martin himself had constructed such sets [Ma1] and later Cohen and Jockusch [CoJ] showed that even Post's simple set is atomless.

Martin conjectured [Ma3] and Robinson proved [Ro1, Theorem 5.3], [Ro2] every coinfinite r.e. set $\boldsymbol{A}$ of low degree has a maximal superset $\boldsymbol{M}$. Robinson's proof combines the maximal set construction with the Kleene recursion theorem [Rg2, Chapter 11] which asserts that for any recursive function $f$ there exists a fixed point $n$ such that $W_{f(n)}=W_{n}$. Like the fixed point theorems of analysis this allows us to prove the existence of certain implicitly defined functions. Specifically if $W_{0} \cap \bar{M}$ is infinite, the recursion theorem allows us to move the markers $\Lambda_{n}$ so that each $\Lambda_{n}$ comes to rest on an element of $W_{0} \cap \bar{A}$, thereby meeting requirement $P_{0}$ of (3.1). (Details can be found in [Ro1] written in the Kleene equational calculus or in [S06] written in a less formal style.) For $A \in \mathcal{E}$ define the lattice of supersets (principal filter) of $A, \mathcal{L}(A)=\{W: W \in \mathcal{E}$ and $A \subseteq W\}$. Soare generalized Robinson's result [S06] by extending the automorphism machinery [S03] to prove

THEOREM 4.1 (SOARE). If a coinfinite r.e set $A$ is low $\left(\operatorname{dg}(A) \in \mathrm{L}_{1}\right)$ then $\mathcal{L}(A) \cong \mathcal{E}$.

Lachlan [La11, Theorem 4] generalized Robinson's result in a different direction by proving that any coinfinite r.e. set which is $\operatorname{low}_{2}\left(\operatorname{dg}(A) \in \mathbf{L}_{2}\right)$ has a maximal superset. (Lachlan's ingenious proof uses the recursion theorem

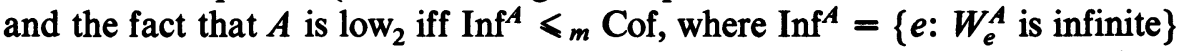
and $\operatorname{Cof}=\left\{e: W_{e} \text { cofinite }\right\}_{-}$Thus, one has certain recursive approximations to determine when $W_{e} \cap \bar{A}$ is infinite. This barely suffices to meet each requirement $\boldsymbol{P}_{e}$.) Shoenfield then gave a remarkably short and elegant proof [Sf7] that every degree $d \in \overline{\mathbf{L}}_{2}$ contains a coinfinite r.e. set $B$ with no maximal (or even $h h$-simple) superset. (Shoenfield proves that for $A \in \mathcal{E}$, if any deficiency set $B$ of $A$ (as defined in \$2) has an $h h$-simple superset then $B$, and hence $A$, are low $_{2}$.)

THEOREM 4.2 (LACHLAN-SHOENFIELD). Let $\mathbb{Q}$ be the class of atomless r.e. sets. Then $\operatorname{dg}(\mathbb{Q})=\bar{L}_{2}$.

It is unknown whether Lachlan's half of this theorem can be combined with Theorem 4.1 to replace $L_{1}$ by $L_{2}$ in the latter, although this seems very difficult. Partial progress for a property resembling ${ } \mathrm{low}_{2}$ may be found in [BeSo, Theorem 4.8].

Besides $\overline{\mathbf{L}}_{0}, \mathbf{H}_{1}, \overline{\mathbf{L}}_{2}$ and the trivial classes it is unknown which other classes $\mathbf{H}_{n}$ and $\overline{\mathbf{L}}_{n}$ are invariant. In particular, is $\bar{L}_{1}$ invariant? As a strong generalization of Theorem 3.6 we propose the following.

CONJECTURE 4.3. Every invariant class $\mathbf{C}$ is closed upwards and contains $\mathbf{H}_{1}$.

Evidence for this conjecture besides Martin's theorem is Theorem 3.2 which asserts that $\left\{0^{\prime}\right\}=\mathbf{H}_{0} \subseteq C$ for any such $\mathbf{C}$. (For any $n>0,0^{\prime} \notin L_{n}$ so $\mathbf{L}_{n}$ cannot be invariant.) Given $\mathcal{C} \subseteq \mathcal{E}$ let $\mathcal{C}^{\#}$ denote the coinfinite r.e. sets with no superset in $\mathcal{C}$, e.g. $\mathbb{Q}=\mathfrak{N}^{\#}$. It seemed plausible to continue the progression of Theorems 3.6 and 4.2 and define $\mathcal{C}_{1}=\Re$ and $\mathcal{C}_{n+1}=\mathcal{C}_{n}^{\#}$. 
However, $\mathcal{C}_{4}=\mathcal{C}_{2}$ and $\operatorname{dg}\left(\mathcal{C}_{3}\right)=\mathbf{H}_{1}[\mathrm{CoJ}]$, so no new invariant classes are obtained. Still unclassified is $e^{\#}$ where $e$ is the class of $r$-maximal sets as defined in $\S 8$, although $\mathbf{H}_{1} \subseteq \operatorname{dg}\left(\bigodot^{\#}\right)$ by Corollary 8.7.

Shoenfield asked [Sf7] whether there are any more invariant classes, and conjectures of Shoenfield and Martin imply at least that every nontrivial invariant class is of the form $\mathbf{H}_{n}$ or $\overline{\mathbf{L}}_{n}$ for some $n$. Recently Lerman and Soare [LeSo2] refuted these conjectures by producing a new invariant class $\mathbf{D}=\operatorname{dg}(\mathscr{D})$, where $\mathscr{D}$ is the class of $d$-simple sets, sets simple with respect to certain r.e. arrays of differences of r.e. sets (d.r.e. sets). The $d$-simple sets are intermediate between simple and $h h$-simple sets and arose in connection with generating automorphisms of $\mathcal{E}$ (see $\S 9$ ). Now $D$ is not of the form $\mathbf{H}_{n}$ or $\overline{\mathbf{L}}_{n}$ for any $n$ because $H_{1} \subseteq D$ and $D$ splits $L_{1}$.

5. Incompleteness and completeness for noninvariant properties. By Theorem 3.2 no property invariant under Aut $\mathcal{E}$ can insure incompleteness, but what of properties not invariant? There is a characterization of low r.e. sets in terms of strong arrays which arose from a consideration of the computational complexity of r.e. sets [S08]. This characterization is the closest known positive solution to Post's original program and Sacks' question [Sa7, Q3] because it does not mention reduction procedures or enumerations of $A$, but it is not as useful in constructions of low r.e. sets as other characterizations which do [S04, Theorem 4.1].

THEOREM 5.1 [S08, THEOREM 2.7]. An r.e. set $A$ is low if and only if there is a recursive function $f$ such that for all $e$,

(i) $(\forall x)\left[D_{x} \subset A\right) \Rightarrow\left[x \in W_{e} \Leftrightarrow x \in W_{f(e)}\right]$, and

(ii) $\left\{x: D_{x} \subseteq \bar{A}\right.$ and $\left.x \in W_{e}\right\}=\varnothing \Rightarrow W_{f(e)}$ is finite.

On the other hand Martin [Ma2] and Lachlan [La7] have discovered an elegant characterization of complete r.e. sets which is useful in applications, for example [GiMr, \$3] and [Sf1]. A set $S$ is effectively simple if there is a recursive function $f$ such that $W_{x} \subseteq \bar{S}$ implies $\left|W_{x}\right|<f(x)$. Martin [Ma2] used the recursion theorem to prove that every effectively simple set $S$ is complete by showing that $K \leqslant T S$ because $C_{K}(x) \leqslant C_{S}(h(x))$, where $h$ is a certain function recursive in $S$ (and $C_{A}$ is the computation function of $A$ as above). The characterization of Martin and Lachlan grew out of the "effectively nonrecursive" sets such as creative sets and effectively simple sets.

THEOREM 5.2 [La7, p. 99]. An r.e. set $A$ is complete iff there is a function $h \leqslant T A$ and a recursive function $g$ such that if $\varphi_{e}[h(z)]=\chi_{A}[h(z)]$ then $W_{g(e, x)} \neq W_{x}$.

This technique can be expanded to yield a certain "maximum degree principle" [JSo2, pp. 613-615] which asserts roughly that when an r.e. set $B$ is constructed recursively in an r.e. set $A$ by the permitting method above, $B$ usually has the same degree as $A$ without explicitly coding $A$ into $B$. This applies to Theorem 3.6.

Post' simple set is effectively simple and therefore complete. (His hypersimple set $H$ may be complete or incomplete [JSo4] depending upon which particular simultaneous enumeration of the r.e. sets is used during the 
construction of $H$. The degree of $H$ under something like the canonical enumeration [K13], which presumably Post intended, is unknown.)

\section{CHAPTER II. THE STRUCTURE, AUTOMORPHISMS}

\section{AND ELEMENTARY THEORY OF THE R.E. SETS}

6. Basic facts and splitting theorems. For a fixed simultaneous enumeration of the r.e. sets define $W_{i} \backslash W_{j}=\left\{x:(\exists s)\left[x \in W_{i, s}-W_{j, s}\right]\right\}$ the elements enumerated in $W_{i}$ before $W_{j}$, and $W_{i} \backslash W_{j}=\left(W_{i} \backslash W_{j}\right) \cap W_{j}$, the elements enumerated in $W_{i}$ and later in $W_{j}$. (The_notation $A \backslash B$ should not be confused with $A-B$ which denotes $A \cap \bar{B}$.) A crucial fact about r.e. sets (which is also true of their generalizations in higher recursion theory) is the reduction principle.

Proposition 6.1 (Reduction Principle). For any $A, B \in \mathcal{E}$ there exist $A_{1}, B_{1} \in \mathcal{E}$ such that $A_{1} \subseteq A, B_{1} \subseteq B, A_{1} \cup B_{1}=A \cup B$ and $A_{1} \cap B_{1}=\varnothing$.

Proof. Let $A_{1}=A \backslash B$ and $B_{1}=B \backslash A$.

There are no interesting ideals in $\mathscr{E}$ because the ideal $\mathscr{F}$ of finite sets is the largest definable ideal, and for any $A \in \mathcal{E}-\mathscr{F}$, the principal ideal $\mathscr{G}(A)=$ $\{W: W \in \mathcal{E}$ and $A \supseteq W\}$ is isomorphic to $\mathcal{E}$. Thus, attention has been focused upon the filters of $\mathscr{E}$ particularly the principal filters $\mathcal{L}(A)$. Among the interesting nonprincipal filters are the simple sets, $h$-simple sets, and $h h$-simple sets (each taken together with the cofinite sets in order to be closed upwards). The principal filters generated by $h h$-simple sets and $r$-maximal sets will be considered in $\$ 7$ and $\$ 8$.

Certain "splitting" theorems arose early in the subject and have played an important role in questions of both structure and decidability of $\mathcal{E}$.

THEOREM 6.2 (FRIEDberg SPLITTING THEOREM [Fr4]). If $A$ is any nonrecursive r.e. set then there exist nonrecursive r.e. sets $B$ and $C$ such that $A=B \cup$ $C$ and $B \cap C=\varnothing$.

Proof. At stage $s+1$ given $x \in A_{s+1}-A_{s}$ find the least $e<s$ such that $x \in W_{e, s}$ and $W_{e, s} \cap B_{s}=\varnothing\left(W_{e, s} \cap C_{s}=\varnothing\right)$ and enumerate $x$ in $B$ (respectively $C$ ). (If no such $e$ exists enumerate $x$ in $C$.) If $W_{e}-A$ is not r.e. then $\left|W_{e} \backslash A\right|=\infty$, so $W_{e} \cap B \neq \varnothing$ and $W_{e} \cap C \neq \varnothing$.

Notice that the proof in fact guarantees

$$
(\forall W \in \mathcal{E})[(W-A) \text { not r.e. } \Rightarrow[(W-B) \text { and }(W-C) \text { not r.e. }]] \text {. }
$$

Later Sacks [Sa2] simultaneously extended both of Friedberg's theorems using a powerful new finite injury method discussed in $\$ 12$.

TheOREM 6.3 (SACKs SplitTing TheOREM). If $A$ is any nonrecursive r.e. set then there exist low r.e. sets $B$ and $C$ such that

(i) $A=B \cup C$ and $B \cap C=\varnothing$; and

(ii) $B$ and $C$ are Turing incomparable.

Then Owings [01, Lemma 1] modified Friedberg's proof above to show

TheORem 6.4 (OWINGs SPlitTing TheOREM). Let $A$ and $D$ be r.e. sets such 
that $A-D$ is not co-r.e. Then there exist r.e. sets $B$ and $C$ (whose indices may be obtained uniformly from those of $A$ and $D$ ) such that

(i) $A=B \cup C$ and $B \cap C=\varnothing$; and

(ii) neither $B-D$ nor $C-D$ is co-r.e.

For any set $S \subseteq \omega$ define $\mathcal{E}_{S}$, the lattice of r.e. sets restricted to $S$, to be $\{W \cap S: W \in \mathcal{E}\}$. (Note that if $A$ is r.e. then $\mathcal{L}(A) \cong \mathcal{E}_{-}$.) For r.e. $W$ let $W_{S}$ denote the set $W \cap S$ of $\mathcal{E}_{s}$. A member $A_{s}$ of $\mathcal{E}_{s}$ is complemented (in $\varepsilon_{S}$ ) if there is an r.e. set $B$ such that $A_{S} \cup B_{S}=S$ and $A_{S} \cap B_{S}=\varnothing$. Friedberg's theorem asserts for $S=\omega$, and Owings' for $S$ co-r.e. (namely $S=\bar{D})$ that every noncomplemented element $A_{S}$ of $\mathcal{E}_{S}$ splits as the disjoint union of two noncomplemented members $B_{S}$ and $C_{S}$ of $\mathcal{E}_{s}$. Morley and Soare [MoSo] obtained a simultaneous generalization of these three theorems using the method of the Sacks Splitting Theorem.

Theorem 6.5 (Morley-Soare Splitting Theorem). For any set $S \leqslant T K$ and any r.e. set $A$, if $A_{s}$ is a noncomplemented member of $\mathcal{E}_{s}$ then there exist r.e. sets $B$ and $C$ (whose indices may be found uniformly from that of $A$ ) such that:

(i) $A=B \cup C$ and $B \cap C=\varnothing$;

(ii) $B_{S}$ and $C_{S}$ are noncomplemented in $\mathcal{E}_{S}$; and

(iii) $B$ and $C$ are Turing incomparable.

7. $h$ h-simple sets. Even though $h$ h-simple sets did not solve Post's problem, they played an important role in the development of the theory of r.e. sets and numerous interesting characterizations of them arose [Y1, Theorem 6] and [Ro4, Theorems 2 and 4]. The most surprising and important of these was discovered by Lachlan [La8] who gave a complete characterization of the principal filters $\mathcal{L}(A)$ generated by the $h h$-simple sets $A$.

TheOREM 7.1 (LACHLAN). For any coinfinite r.e. set $A, A$ is hh-simple iff $\mathcal{L}(A)$ (or equivalently $\mathcal{E}_{-}^{-}$) is a Boolean algebra.

Proof. $\Leftarrow:$ If $A$ is not $h h$-simple choose a disjoint weak array $\left\{W_{f(n)}\right\}_{n \in \omega}$ such that $W_{f(n)} \cap \bar{A} \neq \varnothing$ for all $n$. Then $B_{A}$ is noncomplemented in $\mathcal{E}_{\bar{A}}$ where $B=\cup\left\{W_{n} \cap W_{f(n)}: n \in \omega\right\}$.

$\Rightarrow$ : If $\mathcal{L}(A)$ is not a Boolean algebra then there exists an r.e. $B \supseteq A$ such that $B-A$ is not co-r.e. Now by repeatedly applying Owings Splitting Theorem B may be split to produce a disjoint r.e. array of r.e. sets $\left\{C_{n}\right\}_{n \in \omega}$ such that $C_{n} \cap \bar{A} \neq \varnothing$ for all $n$. Split $B$ into $C_{0} \cup D_{0}$, then split $D_{0}$ into $C_{1} \cup D_{1}$, and so forth. The array $\left\{C_{n}\right\}_{n \in \omega}$ may be converted into a disjoint weak array of finite sets $\left\{\hat{C}_{n}\right\}_{n \in \omega}$, such that $\hat{C}_{n} \cap \bar{A} \neq \varnothing$, by enumerating $x$ in $\hat{C}_{n}$ at stage $s+1$ only if $\hat{C}_{n, s} \subseteq A_{s}$ and $x=(\mu y)\left[x \in C_{n, s}-A_{s}\right]$.

An infinite set $S$ is hyperhyperimmune (hh-immune) if there is no disjoint weak array of finite sets $\left\{F_{n}\right\}_{n \in \omega}$ such that $F_{n} \cap S \neq \varnothing$ for all $n$. (For $S \leqslant T K$ the condition "finite" is redundant.) The above proof actually establishes

Theorem 7.2 (LACHLAN). If $A, B \in \mathcal{E}$ and $A-B$ is hh-immune then $A-B$ is co-r.e. 
Lachlan then wondered exactly which Boolean algebras could be realized as $\mathcal{L}^{*}(A)$ for some $A \in \mathcal{E}$, for example the countable atomless Boolean algebra or any recursive Boolean algebra.

THEOREM 7.3 (LACHLAN). There exists an hh-simple set $A$ such that $\mathcal{L}^{*}(A)$ is an atomless Boolean algebra.

SKETCH OF PROOF. In the maximal set construction (Theorem 3.3) we could have pictured the markers $\left\{\Lambda_{e}\right\}_{e \in \omega}$ as fixed "boxes" or "windows" and the elements $m_{e}^{s}$ as moving (downward) along the markers and dropping into the set $M$. Let $2^{<\omega}$ denote all finite sequences of 0 's and l's. For each $\sigma \in 2^{<\omega}$ we have a nonmovable marker $\Lambda_{\sigma}$. These are given an $\omega$ ordering by some recursive function $f: 2^{<\omega} \rightarrow \omega$ such that $\operatorname{lh}(\sigma)<\operatorname{lh}(\tau)$ implies $f(\sigma)<f(\tau)$, where $\ln (\sigma)$ denotes the length of $\sigma$. An element $x$ can move from $\Lambda_{\tau}$ to $\Lambda_{\sigma}$ only if $f(\sigma)<f(\tau)$. Each $\Lambda_{\sigma}$ marks an integer $m_{\sigma}^{s}$ at the end of stage $s$, and $\bar{A}=\left\{m_{\sigma}: \sigma \in 2^{<\omega}\right\}$ where $m_{\sigma}=\lim _{s} m_{\sigma}^{s}$. For each $\sigma$ define the "cone" $C_{\sigma}=\left\{m_{\tau}: \sigma \subseteq \tau\right\}$, where $\sigma \subseteq \tau$ denotes that $\sigma$ is an initial segment of $\tau$. It clearly suffices to meet for each $e$ the requirements $P_{e}: \operatorname{lh}(\sigma)=e \Rightarrow(\forall i \leqslant$ $e)\left[W_{i} \cap C_{\sigma}={ }^{*} \varnothing\right.$ or $\left.C_{\sigma} \subseteq^{*} W_{i}\right]$, and $N_{e}: A \cup C_{e}$ is r.e.

Surprisingly these requirements are automatically satisfied by simply performing at stage $s+1$ the usual strategy for $P_{e}$ within each cone $C_{\sigma}^{s}=$ $\left\{m_{\tau}^{s}: \sigma \subseteq \tau\right\}$, such that $\operatorname{lh}(\sigma)=e$.

A $\Sigma_{3}$-relation $R$ on $\omega \times \omega$ is one for which there is a recursive relation $S$ on $\omega^{5}$ such that $R(x, y) \equiv(\exists u)(\forall v)(\exists w) S(x, y, u, v, w)$. A $\Sigma_{3}$-lattice $\mathbb{Q}=\langle A,\langle, \cap, \cup\rangle$ is one for which there exist binary recursive functions $U$ and $I$ and a $\Sigma_{3}$-relation $S$ such that if $A=\left\{a_{x}\right\}_{x \in \omega}$ then $a_{x} \cup a_{y}=a_{U(x, y)}$, $a_{x} \cap a_{y}=a_{I(x, y)}$ and such that $S=\left\{\langle x, y\rangle: a_{x} \leqslant a_{y}\right\}$. (Note that $a_{x} \leqslant a_{y}$ is a $\Sigma_{3}$-relation of $x$ and $y$.) For any $A \in \mathcal{E}, \mathcal{L}^{*}(A)$ is a $\Sigma_{3}$-lattice because the relation $\left\{\langle x, y\rangle: W_{x} \subseteq{ }^{*} W_{y}\right\}$ is easily seen to be $\Sigma_{3}$ (see [Rg2, Chapter 14]).

By a more complicated construction than that for Theorem 7.3, Lachlan uses a fixed $\Sigma_{3}$-relation to "prune" branches from the full binary tree $2^{\omega}$ and to prove,

THEOREM 7.4 (LACHLAN). If $\mathscr{Q}$ is any $\Sigma_{3}$-Boolean algebra then there is an hh-simple set $A$ such that $\mathcal{L}^{*}(A) \cong \mathbb{Q}$.

This completely characterizes the principal filters of $h h$-simple sets, although their automorphism types under Aut $\mathscr{E}$ remain largely unknown (see \$9). Alton [A1] has applied Lachlan's Theorem together with results of Spector on inductive definitions [Sp2] to show that an $\alpha$ th order maximal set exists iff $\alpha$ is a recursive ordinal. (An $\alpha$ th order maximal set is a coatom of $\mathcal{E}^{\alpha}$, the lattice obtained from $\mathcal{E}$ by factoring $\alpha$ times by the filter generated by coatoms.)

Finally, let $\mathcal{H C}$ denote the lattice of cofinite and $h h$-simple sets. By Theorem 7.2, $\mathfrak{H}^{*}$ is a relatively complemented distributive lattice and hence has a decidable elementary theory by Ershov's result [E1] that all complete extensions of the elementary theory of relatively complemented distributive lattices are decidable. (A lattice $L$ is relatively complemented if whenever $a, b, c \in L$ and $a \leqslant b \leqslant c$ there exists $d \in C$ with $b \cap d=a$ and $b \cup d=$ c.) 
8. Major subsets and $r$-maximal sets. Consider those lattices $\mathfrak{L} \cong \mathfrak{L}^{*}(A)$ for some $A \in \mathcal{E}$. Lachlan's Theorem 7.4 gives a complete characterization for those which are Boolean algebras. Little is known about the remaining lattices $\mathcal{L}$ except that $\mathscr{E}^{*}$ is embedded in each and so the structure of $\mathcal{L}$ is apparently at least as complicated as that of $\mathcal{E}^{*}$ itself.

Proposition 8.1. If $A \in \mathcal{E}$ and $\mathcal{L}^{*}(A)$ is not a Boolean algebra then $\mathcal{E}$ is embeddable in $\mathfrak{L}(A)$.

Proof. Clearly $\bar{A}$ is infinite. By Theorem 7.1 $A$ is not $h h$-simple so there is a disjoint weak array $\left\{W_{f(n)}\right\}_{n \in \omega}$ such that $W_{f(n)} \cap \bar{A} \neq \varnothing$ for all $n$. Let $W_{g(x)}=A \cup \cup\left\{W_{f(n)}: n \in W_{x}\right\}$. Then the mapping $W_{x} \rightarrow W_{g(x)}$ from $\mathcal{E}$ to $\mathfrak{L}(A)$ is $1: 1$ and preserves inclusion.

In addition Shore has noted that for every $A \in \mathcal{E}, \mathfrak{L}^{*}(A) \times \mathcal{L}^{*}(K) \cong$ $\mathfrak{L}^{*}(K)$, and that $\mathfrak{L}^{*}(A) \cong \mathfrak{L}^{*}(K)$ implies that $A$ is high. Shore has also shown that $\mathcal{L}^{*}(A) \times \mathcal{E}^{*} \cong \mathfrak{L}^{*}(B) \times \mathcal{E}^{*}$ implies either that $\mathcal{L}^{*}(A) \cong \mathscr{L}^{*}(B)$ or $\mathfrak{L}^{*}(A) \cong \mathfrak{L}^{*}(B) \times \mathcal{E}^{*}$.

At the other extreme from Boolean algebras are those lattices containing no complemented elements (except for the trivial elements 0 and 1). An r.e. set $A$ is $r$-maximal if $\bar{A}$ is infinite and $\mathfrak{L}^{*}(A)$ contains no nontrivial complemented elements, or equivalently if $\bar{A}$ is $r$-cohesive namely infinite and there is no recursive set $R$ such that $R \cap \bar{A} \neq \neq^{*} \varnothing$ and $\bar{R} \cap \bar{A} \neq{ }^{*} \varnothing$. Such sets exist, and they may or may not have maximal supersets. (If an $r$-maximal set $A$ has a maximal superset $M$ then by Proposition 6.1, $M$ must be unique.) The easiest construction of $r$-maximal sets with maximal supersets is by using the major subsets of Lachlan [La8, p. 29], which play a crucial role in the decision procedure of $\S 10$. For $A, B \in \mathcal{E}, B$ is a major subset of $A\left(B \subset_{m} A\right)$ if $B \subset_{\infty} A$ and for all $W \in \mathcal{E}$,

$$
A \cup W={ }^{*} \omega \Rightarrow B \cup W={ }^{*} \omega .
$$

THEOREM 8.2 (LACHLAN). If $A$ is a nonrecursive r.e. set then $A$ contains $a$ major subset $B$.

SKETCH OF PROOF. The proof is very similar to the maximal set construction except that the markers $\left\{\Lambda_{e}\right\}_{e \in \omega}$ come to rest on $A-B$ and seek to maximize their $e$-states with respect to $\left\{V_{n}\right\}_{n \in \omega}$ in place of $\left\{W_{n}\right\}_{n \in \omega}$ where $V_{n, s}=\left\{x: x \in W_{n, s}\right.$ and $\left.x \leqslant f(n, s)\right\}$, for $f(n, s)=(\mu x)\left[x \notin W_{n, s} \cup A_{s}\right]$. (Note that $V_{n}=W_{n}$ if $W_{n} \supseteq \bar{A}$ and $V_{n}$ is finite otherwise.) Now for example, if $\bar{A} \subseteq W_{0}$ then $V_{0}=W_{0},\left|V_{0} \searrow A\right|=\infty$ (because $\bar{A}$ is not r.e.), and hence all markers $\Lambda_{e}$ come to rest on elements of $V_{0}$, so $A-B \subset V_{0}$.

Proposition 8.3. If $B \subset_{m} A$ and $A$ is $r$-maximal then $B$ is $r$-maximal.

Proof. For any recursive set $R$ either $\bar{A} \subseteq{ }^{*} R$ or $\bar{A} \subseteq{ }^{*} \bar{R}$, say the former. Then $\bar{B} \subseteq{ }^{*} R$ because $B \subset_{m} A$.

Thus, if $A$ is a maximal set then any major subset is a proper $r$-maximal set. Furthermore, no $r$-maximal set can also be $h h$-simple unless $A$ is maximal.

Lachlan [La8, Theorem 8], [La12, p. 300] and Robinson [Ro4, Theorem 6] also independently constructed $r$-maximal sets with no maximal superset. Robinson noted that it clearly suffices to construct a "tower" of r.e. sets 
$\left\{T_{n}\right\}_{n \in \omega}$ such that $A \subset_{\infty} T_{0} \subset_{\infty} T_{1} \subset_{\infty} \ldots$, and such that for all $n, W_{n} \subseteq$ $T_{n}$ or $\bar{A} \subseteq{ }^{*} W_{n}$. (The sequence $\left\{T_{n}\right\}_{n \in \omega}$ need not be r.e.) A planar version of the Friedberg maximal set construction easily produces this tower. Such a construction with certain additional properties necessary for the extended decision procedure of $\$ 10$ is carried out by Lerman and Soare [LeSo1, Theorem 2.15]. (Lachlan's construction [La8, Theorem 8] actually produces an $r$-maximal set with no dense simple superset, while Robinson's produces an atomless $r$-maximal set which is dense simple.)

Major subsets may be used to produce sets which fail to have $r$-maximal or $h h$-simple supersets. A coinfinite r.e. set $A$ is strongly hypersimple (sh-simple) if there is no r.e. array $\left\{W_{f(n)}\right\}_{n \in \omega}$ of disjoint r.e. sets such that $\omega=\cup_{n} W_{f(n)}$ and $\bar{A} \cap W_{f(n)} \neq \varnothing$ for all $n$. Clearly, every $r$-maximal or $h h$-simple set is sh-simple.

Proposition 8.4. Let $A, B \in \mathcal{E}, B \subset_{m} A, f$ a $1: 1$ recursive function with range $A$ and $\hat{B}=f^{-1}(B)$. Then $\hat{B}$ is h-simple but has no sh-simple superset.

Proof. Clearly $\hat{B}$ must be $h$-simple since if $\hat{B}$ is not $h$-simple as witnessed by $\left\{D_{g(n)}\right\}_{n \in \omega}$ then from $f\left(\left\{D_{g(2 n)}\right\}_{n \in \omega}\right)$ we can choose a recursive set $R$ such that $R \subseteq A$ and $R$ splits $A-B$, so $\bar{R}$ violates $B \subset_{m} A$.

Now suppose $\hat{B} \subset D \subset_{\infty} \omega$, for $D$ r.e. Then $A-f(D)$ is not co-r.e. so by the proof of Theorem 7.1 there is a disjoint r.e. array $\left\{W_{g(n)}\right\}_{n \in \omega}$ with union $A$ and such that $W_{g(n)} \cap(A-f(D)) \neq \varnothing$ for all $n$. Let $W_{h(n)}=f^{-1}\left(W_{g(n)}\right)$. Then $\left\{W_{h(n)}\right\}_{n \in \omega}$ is a disjoint r.e. array of r.e. sets with union $\omega$ and such that $W_{h(n)} \cap \bar{A} \neq \varnothing$ for all $n$. Hence, $D$ is not sh-simple.

R. W. Robinson has shown [Ro5, p. 345] that $\hat{B}$ must be dense simple. Hence $\hat{B}$ and $B$ must be high. Stob [St] proved that $\hat{B}$ must be a major subset of some $C \subset_{\infty} \omega$.

Define the relation $\equiv_{r}$ on $r$-maximal sets by $A \equiv_{r} B$ iff $A \cap B$ is $r$ maximal. It is easily verified that $\equiv_{r}$ is an equivalence relation and that $A \equiv{ }_{r} B$ iff $A \cup B \neq \neq^{*} \omega$. For any maximal set $M$ the $r$-maximal subsets of $M$ constitute an equivalence class under $\equiv_{r}$ consisting precisely of the major subsets of $M$. Similarly there are infinitely many equivalence classes of atomless $r$-maximal sets.

For any atomless $r$-maximal set $A, \mathcal{L}^{*}(A)$ is densely ordered by the Owings Splitting Theorem. Thus, one might hope that $\mathfrak{L}^{*}(A) \cong \mathfrak{L}^{*}(B)$ for any two such sets, but this is false [LeSo1]. These isomorphism types of $\mathcal{L}^{*}(A)$ as well as the automorphism types of $r$-maximal sets $A$ under Aut $\mathscr{E}$ remain unclassified.

As for the $r$-maximal sets with maximal superset, define a difference of r.e. sets (d.r.e. set) $D=A-B$ to be pure d.r.e. if it is infinite but contains no infinite r.e. or co-r.e. subsets. (It is easily seen that $D$ is pure d.r.e. iff $D=A-B$ for some $A$ and $B$ such that $B C_{m} A$.) It is an open question whether $\mathcal{E}_{D} \cong \mathcal{E}_{E}$ for any two pure d.r.e. sets $D$ and $E$. Resolution of these questions will require a much closer analysis of which finite unions of d.r.e. sets are r.e. This is a major theme of Chapter II. (Ershov [E2] has considered hierarchies generated by finite and even infinite unions of d.r.e. sets.)

Major subsets are also useful in studying degrees of r.e. sets, since Lerman [Le3] combined the constructions of Martin (Theorem 3.6) and Lachlan 
(Theorem 8.2) to prove

TheOREM 8.5 (LeRMAN). For any nonrecursive r.e. set $A$ and degree $\mathbf{d} \in \mathbf{H}_{1}$ there exists an r.e. set $B \in \mathrm{d}$ such that $B \subset_{m} A$.

The converse follows from the remarks after Proposition 8.4, or by [J4].

TheOREM 8.6 (Robinson). If $B \subset_{m} A$ then $B$ has high degree.

COROLlary 8.7 (SHORE). If $\mathcal{C}$ is a class of coinfinite sets which is closed upwards among the coinfinite sets (i.e. $A \in \mathcal{C}$ and $A \subset B \subset \subset_{\infty}$ w imply $B \in \mathcal{C}$ ) and $\bigodot^{\#} \neq \varnothing$ then $\mathbf{H}_{1} \subseteq \mathrm{dg}\left(\bigodot^{\#}\right)$.

Proof. Let $\mathcal{C}^{\#}$ be as in $\S 4$. If $A \in \mathcal{C}^{\#}$ and $B \subset_{m} A$ then $B \in \mathcal{C}^{\#}$, because any $S \in \mathcal{C}$ such that $B \subseteq S$ must satisfy $\bar{S} \subseteq^{*} A-B$ since $A \in \bigodot^{\#}$, but this violates $B \subset_{m} A$. Now apply Theorem 8.5.

In particular, $\mathbf{H}_{1} \subseteq \operatorname{dg}\left(C^{\#}\right)$ for all classes $\mathcal{C}$ of simple sets such as $r$-maximal, dense simple, or sh-simple, etc. Theorem 8.5 and Corollary 8.7 are further evidence for Conjecture 4.3. For the three classes above, except the $r$-maximal sets, Martin [Ma3] has shown that $\operatorname{dg}\left(\bigodot^{\#}\right)=\mathbf{H}_{\mathbf{1}}$. Still unclassified are the degrees of simple sets $A$ with no $r$-maximal major subset as defined below.

Two special kinds of major subsets arise in the study of automorphisms and decision procedures for $\mathcal{E}$, namely the $r$-maximal major subsets and the small major subsets. A major subset $B C_{m} A$ is an $r$-maximal major $(r m)$ subset of $A$ if $A-B$ is $r$-cohesive. By Theorem 7.2 if $B C_{m} A$ then $A-B$ cannot be $h h$-immune, so $r$-cohesiveness is the "thinnest" property $A-B$ can hope to possess. Many simple sets such as $r$-maximal sets possess $r m$ subsets but some do not. The question of whether atomless $h h$-simple sets can possess them was answered in [LeSo1] as a necessary ingredient in the extended decision procedure of $\$ 10$. This led to a classification [LeShSo] of those nonrecursive r.e. sets $A$ which possess an $r m$ subset as precisely those such that $\bar{A}$ has a $\Delta_{3}$-preference function, roughly a $\{0,1\}$ valued function $f$ whose graph is $\Sigma_{3}$ (and hence $\Delta_{3}$ ) and which for each recursive set $R_{i}$ specifies $R_{i}$ or $R_{i}$ as infinite on $\bar{A}$ and thus to be preferred in the construction of $B \subset_{m} A$.

COROLlARY 8.8 (LERMAN-SHORE-SOARE). There exist atomless hh-simple sets $A$ and $B$ such that $A$ has an rm subset but $B$ does not.

At the other extreme are major subsets $B \subset_{m} A$ such that $A-B$ is "large," i.e. $B$ is "small," a notion invented by Lachlan [La9] for his decision procedure.

Definition 8.9. (i) If $A, B \in \mathcal{E}$ and $B \subset A \subset \subset_{\infty} \omega$, then $B$ is small in $A$ $\left(B \subset_{s} A\right)$ if for all $U, V \in \mathcal{E}$,

$$
V \supseteq U \cap(A-B) \Rightarrow(U-A) \cup V \text { is r.e., }
$$

(ii) $B$ is small if $B \subset_{s} A$ for some $A \subset_{\infty} \omega$.

The intuition is that $B$ is sufficiently smaller than $A$ so that any $V$ satisfying the hypothesis of (8.1) must include enough of $U$ so that the union of the d.r.e. set $(U-A)$ with $V$ is r.e. Notice that $\varnothing \subset_{s} A$ for every r.e. $A \subset_{\infty} \omega$ (because $(U-A) \cup V=U \cup V)$, and for $A$ nonrecursive no $B={ }^{*} A$ is 
small in $A$. (If so set $U=\omega$ and $V=A-B$ implying that $\bar{A}$ is r.e.) The name "small" was given by $M$. Stob [St] who observed that for r.e. sets $A \subset B \subset C \subset \subset_{\infty} \omega$, if either $A \subset_{s} B$ or $B \subset_{s} C$ then $A \subset_{s} C$. (Thus, on $\mathcal{E}$ the small sets are closed downward under inclusion and $C_{s}$ is transitive.) Stob also observed that for r.e. sets $A \subset_{\infty} C \subset_{\infty} \omega$, if $A$ is not recursive and $C-A$ is not co-r.e. then there exists an r.e. $B$ such that $A \subset B \subset C, A \not \ell_{s} B$ and $B \not Z_{s} C$. (Hence, no notion of $A$ being "close" to $C$ can force all intermediate sets $B$ to be small in $C$.) The following extension of Theorem 8.2 is a necessary ingredient [La9, Theorem 3] for Lachlan's decision procedure of $\$ 10$.

THEOREM 8.10 (LACHLAN). If $A$ is a nonrecursive r.e. set then $A$ contains $a$ major subset $B$ small in $A$ (called a small major subset and written $B \subset_{s m} A$ ).

The $r$-maximal major subsets in general lie at the opposite extreme from the small major subsets because if $A$ is simple then no $B \subset_{m} A$ can satisfy both $B \subset_{r m} A$ and $B \subset_{s m} A$. See [LeShSo, Proposition 1.7], except in the trivial case when $A$ is $r$-maximal so $B \subset_{m} A$ implies $B \subset_{r m} A$.

9. Automorphisms of $\mathcal{E}$. Most known information on automorphisms of $\mathcal{E}$ and $\mathcal{E}^{*}$ may be found in [Rg2, Chapter 12] and [So3]. Every automorphism of $\mathcal{E}^{*}$ is induced by an automorphism of $\mathcal{E}$ and there are $2^{\kappa_{0}}$ automorphisms of $\mathcal{E}^{*}$ (Lachlan). In [S03] a powerful new method is introduced for generating automorphisms of $\mathcal{E}$ and it is used to prove,

THEOREM 9.1 (SOARE). If $A$ and $B$ are maximal sets then there is an automorphism $\Phi$ of $\mathcal{E}$ such that $\Phi(A)=B$.

Indeed it is shown that the group Aut $\mathcal{E}^{*}$ is $k$-ply transitive on its coatoms (i.e. any $k$ distinct coatoms of $\mathscr{E}^{*}$ may be carried by an automorphism to any other $k$ distinct coatoms). This method for generating automorphisms has been further developed and applied in Theorem 3.2 [So7], Theorem 4.1 [S06] and by Stob [St] but it remains complicated and not widely understood.

An important question is the study of which properties of r.e. sets are invariant (under Aut \&). Using an earlier "finite-injury" priority argument Martin proved that hypersimplicity is not invariant and later Stob [St] using the full automorphism machinery proved that dense simplicity is also not invariant. The invariance of strong hypersimplicity is unknown. The properties of simplicity, maximality, $r$-maximality, and $h h$-simplicity (by Theorem 7.1) are clearly invariant.

Probably the most important application of automorphisms will be to find complete sets of invariants (in the sense of Felix Klein) for classifying the automorphism types of members of $\mathcal{E}$. The orbit of $A$ is the class of all r.e. sets $B$ automorphic to $A$ in the sense that $B=\Phi(A)$ for some $\Phi \in$ Aut $\mathcal{E}$. The only known definable orbits are the maximal sets and the infinite, coinfinite recursive sets.

Other natural candidates to examine are the $h h$-simple sets (which resemble maximal sets) and the low sets (which resemble recursive sets). It seemed likely that Theorem 9.1 could be generalized by showing that for $h h$-simple sets $A$ and $B, \mathfrak{L}^{*}(A) \cong \mathfrak{L}^{*}(B)$ implies $A$ automorphic to $B$, but this proved 
false even for atomless $h h$-simple sets by Corollary 8.8 . This suggests that for each $h h$-simple set $A$ there will be infinitely many nonautomorphic sets $\left\{B_{n}\right\}_{n \in \omega}$ such that $\mathcal{L}^{*}(A) \cong \mathcal{L}^{*}\left(B_{n}\right)$, and no natural condition for classifying their orbits has arisen.

A second promising class for classification by automorphisms is that of the coinfinite low r.e. sets $A$ because $\mathcal{L}(A) \cong \mathcal{E}$ by Theorem 4.1. It is natural to conjecture that any two low simple sets are automorphic, but Lerman and Soare [LeSo2] refuted this by introducing the notion of $d$-simplicity (simplicity with respect to certain r.e. arrays of d.r.e. sets) and proving that there are low simple sets $A$ and $B$ such that $A$ is $d$-simple and $B$ is not. The notion of $d$-simplicity is a weaker version of a certain "covering property" [So3, Theorem 2.2] sufficient for generating automorphisms, and possessed by maximal sets. It is hoped that stronger versions of $d$-simplicity will be successful in classifying orbits.

Definition 9.2. A coinfinite r.e. set $A \in \mathcal{E}$ is $d$-simple if for all $X \in \mathcal{E}$ there exists an r.e. $Y \subseteq X$ such that

(i) $X \cap \bar{A}=Y \cap \bar{A}$, and

(ii) $(\forall Z \in \mathcal{E})[(Z-X)$ infinite $\Rightarrow(Z-Y) \cap A \neq \varnothing]$.

Notice that (ii) asserts that $A$ is simple with respect to the r.e. array of d.r.e. sets $\left\{\left(W_{e}-Y\right)\right\}_{e \in \omega}$. It is easy to check that every $d$-simple set $A$ is simple (set $X=\varnothing$ ) and every $h h$-simple set is $d$-simple (by Theorem 7.1), but neither converse holds. It is also easy to see that no $d$-simple set is small but the converse fails. Lerman and Soare [LeSo2] have shown that the class $\mathscr{D}$ of $d$-simple sets is the first known definable and invariant class $\mathcal{C} \subseteq \mathcal{E}$ such that $\operatorname{dg}(\mathcal{C})$ splits $\mathbf{L}_{\mathbf{1}}$.

THEOREM 9.3 (LERMAN-SOARE). There exists a low degree a such that every r.e. set of degree $<\mathrm{a}$ is small (and hence not d-simple).

(Thus the degrees $\mathbf{N}$ containing nonsmall r.e. sets like $\operatorname{dg}(\mathscr{Q})$ satisfy $H_{1} \subseteq \mathbf{N}$ and $\mathbf{N}$ splits $L_{1}$. It is unknown whether $\mathbf{N}=\operatorname{dg}(\mathscr{D})$.)

The difficulty in attempting to produce a $d$-simple set $A$ below a fixed nonrecursive r.e. degree by the permitting method [Y2] is that the properties of $d$-simplicity and smallness involve d.r.e. sets (not merely r.e. sets) and are not compatible with the usual permitting methods. This suggests that a proof of Conjecture 4.3 will be difficult, probably nonuniform, and may involve some new coding method.

Open questions include the following. Are any two low $d$-simple sets automorphic? If not, what conditions can be found for classifying orbits of low simple sets? If $\mathbf{a}, \mathbf{b} \in \mathbf{L}_{1}-\mathbf{L}_{0}$ do there always exist simple sets $A \in \mathbf{a}$ and $B \in \mathbf{b}$ such that $A$ is automorphic to $B$ ? If the structures $\langle\mathcal{E}, A\rangle$ and $\langle\mathcal{E}, B\rangle$ are elementarily equivalent (i.e, $A$ and $B$ satisfy all the same formulas with one free variable in the language of $\S 10$ ) then is $A$ automorphic to $B$ ?

A class $\mathcal{C}^{*} \subseteq \mathcal{E}^{*}$ is an automorphism basis (for $\mathcal{E}^{*}$ ) if every $\Phi \in$ Aut $\mathcal{E}^{*}$ is uniquely determined by its action on $\mathcal{C}^{*}$. Shore proves [Sh2] and [Sh3] that if $\mathcal{C} \subseteq \mathcal{E}$ is any nonempty class closed under recursive permutations then $\mathcal{C}^{*}$ is an automorphism basis. He also proves that if $\mathcal{C}^{*}=\Re^{*}$ or if $\mathcal{C}^{*} \subseteq \mathcal{S}^{*}$ then there is an automorphism of (the lattice generated) by $\mathcal{C}^{*}$ which does not extend to any of $\mathcal{E}^{*}$. These results led Shore to define [Sh2] a new class of 
nonsimple sets. An r.e. set $A$ is nowhere simple if for every r.e. $W$ such that $A \subset_{\infty} W$, there is an infinite r.e. $V \subseteq W-A$. Shore proves that every r.e. degree contains a nowhere simple set, and that every r.e. set is the disjoint union of nowhere simple sets. In view of the resemblance of recursive sets to nowhere simple sets the major open question for the latter is whether $\mathcal{L}^{*}(A) \cong \mathcal{E}^{*}$ for every nowhere simple set.

10. The elementary theory of $\mathcal{E}$. One of the major open questions on $\mathcal{E}^{*}$ is the decidability of its elementary theory. Lachlan [La8] proved that the theories of $\mathcal{E}$ and $\mathcal{E}^{*}$ are equi-decidable and gave [La9] a decision procedure for the $\vec{\forall} \vec{\exists}$-sentences of the theory of $\mathcal{E}^{*}$. Lerman and Soare [LeSo1] extended this by adding additional one place relation symbols Max and $H h s$ distinguishing the maximal and $h h$-simple sets. The aim is to add enough additional predicates to give a decision procedure first for the $\vec{\exists} \vec{\forall} \vec{\exists}$-sentences, and then perhaps all sentences.

Let $\mathbb{Q}$ denote the Boolean algebra generated by $\mathcal{E}$, i.e. all finite unions of d.r.e. sets. Lachlan considers [La9] a first order language $L$ which has function symbols $U, \cap, '$, and constant symbol 0 to be interpreted in a Boolean algebra as join, meet, complement and least element respectively, and which has a unary predicate symbol $E(x)$ to be interpreted over $\mathbb{Q}^{*}$ as " $x \in \mathcal{E}^{*}$. An $\vec{\forall} \vec{\exists}$-sentence in this language is one of the form $\left(\forall x_{1}\right) \cdots\left(\forall x_{n}\right)\left(\exists y_{1}\right) \cdots\left(\exists y_{m}\right) P\left(x_{1}, \ldots, x_{n} ; y_{1}, \ldots, y_{m}\right)$ with $P$ quantifier free. Lachlan gives an algorithm for deciding which $\vec{\forall} \vec{\exists}$-sentences are true in $Q^{*}$ when the quantifiers range over $\mathcal{E}^{*}$.

Lachlan easily reduced the $\vec{\forall} \vec{\exists}$-decision problem to the following. Given finite lattices $L, L_{1}, \ldots, L_{k}$, such that each $L_{i}$ is a refinement of $L$, when is it true that for all sublattices $\mathcal{E}$ of $\mathcal{E}^{*}$ such that $\mathcal{L} \cong L$, there exists a sublattice $\mathfrak{L}^{\prime}$ of $\mathcal{E}^{*}$ such that one of the following diagrams commutes.

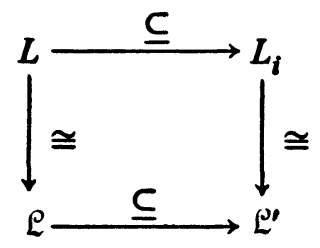

FIGURE 1

First for each finite "separated" lattice $\hat{L}$ Lachlan produces a "canonical realization" $\hat{\mathfrak{L}} \subseteq \mathcal{E}^{*}, \hat{\mathrm{L}} \simeq \hat{L}$. These canonical realizations provide necessary conditions for the diagram of Figure 1 to commute. Secondly, Lachlan proves a refinement theorem (refining the given $\mathfrak{L}$ to produce $\mathfrak{L}^{\prime}$ ) which shows that the necessary conditions are also sufficient. To construct the canonical realizations Lachlan needs only the following facts about $\mathcal{E}$ :

(1) the Reduction Principle (Proposition 6.1);

(2) for any infinite $A \in \mathcal{E}, \mathcal{E}_{A} \simeq \mathcal{E}$;

(3) there is an infinite, coinfinite recursive set;

(4) there is a maximal set (Theorem 3.3);

(5) the Friedberg Splitting Theorem (Theorem 6.2) with condition (6.1);

(6) the small major subset theorem (Theorem 8.10). 
For example consider the lattices of sets $L_{1}$ and $L_{2}$ shown in Figures 2 and 3 , where it is understood that $x_{0}$ is the greatest element and that $E(x)$ holds only of the circles or squares labelled $x_{i}, 1<i<4$, and their unions (and not of the annuli $x_{0}-x_{1}, x_{1}-x_{2}$, and so on). (By the reduction principal, it suffices to consider lattices of sets where the circles $x_{i}$ do not overlap, namely either $x_{i} \cap x_{j}=\varnothing$, or $x_{i} \subseteq x_{j}$ or $x_{j} \subseteq x_{i}$.)

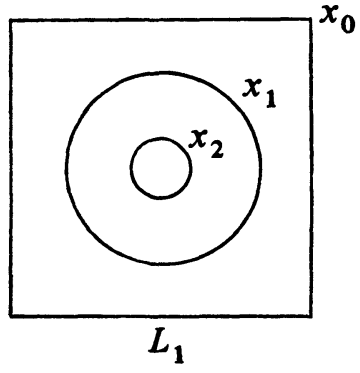

FigURE 2

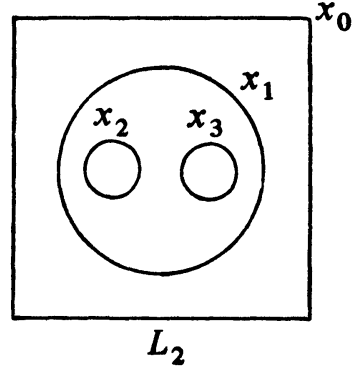

FIGURE 3

To construct a canonical realization $\varrho_{1}$ of $L_{1}$, Lachlan takes $A_{0}=\omega, A_{1}$ a maximal set, and $A_{2} \subset_{s m} A_{1}$. For $L_{2}$ Lachlan applies Friedberg splitting to split $A_{2}$ into $A_{3}$ and $A_{4}$. Let $\varrho_{1}=\left\{A_{0}, A_{1}, A_{2}\right\}$ and $\mathscr{L}_{2}=\left\{A_{0}, A_{1}, A_{3}, A_{4}\right\}$. Notice that $\mathfrak{L}_{1}$ rules out for example, any r.e. set $W$ which might either split $A_{0}-A_{1}$ or such that $\bar{A}_{1} \subseteq W$ but $A_{1}-A_{2} \underline{Z}^{*} W$. Thus these examples rule out as false a large class of $\vec{\forall} \vec{\exists}$ sentences. Taking small major subsets instead of ordinary major subsets produces examples which rule out even more such sentences.

Lachlan defines a notion of "characteristic" for finite lattices which well orders them. Lachlan's refinement theorem [La9, Theorem 4] asserts that either $\mathcal{L}^{\prime} \subseteq \mathcal{E}^{*}$ exists such that the diagram in Figure 1 commutes or else a counterexample has already been found among the canonical realizations of a certain finite effectively determined set of lattices of smaller characteristic than that of $L$. This is the essence of his decision procedure.

Lachlan's refinement theorem itself is quite powerful and immediately yields among other things the Owings Splitting Theorem. A similar method combined with the major subset construction yields,

Theorem 10.11 (Lachlan Interpolation Theorem [La8, p. 35 T2]). For any r.e. sets $B \subseteq A$ such that $A-B$ is not co-r.e., there exists an r.e. $C$ (which can be found effectively from $A$ and $B$ ) such that $B \subseteq C$ and $C \subseteq_{m} A$. low.)

(Of course, we cannot strengthen the conclusion to $B \subseteq_{m} C$ since $B$ may be

The next step in the decision procedure for $\mathcal{E}^{*}$ is to consider the $\vec{\exists} \vec{\forall} \vec{\exists}$ sentences of $L$, namely those of the form $(\exists \vec{x})(\forall \vec{y})(\exists \vec{z}) P(\vec{x}, \vec{y}, \vec{z})$, where $P$ is quantifier free. The most reasonable attack on this problem seems to be to add new predicates to the language $L$ such as $\operatorname{Max}(x)$ and $H h s(x)$ which are to be interpreted in $\mathcal{E}^{*}$ as " $x$ is maximal" and " $x$ is $h h$-simple." Lerman and Soare [LeSo1] have given a decision procedure for the $\vec{\forall} \vec{\exists}$-sentences in this expanded language $L^{+}$, where the quantifiers range over $\mathcal{E}^{*}$. 
The idea is to construct canonical realizations and to prove a refinement theorem as in Lachlan's proof, but each of these steps is much more complicated than in the former case. First note that many new statements become $\vec{\forall} \vec{\exists}$ in this expanded language $L^{+}$such as "there exists an atomless $h h$-simple set with an $r$-maximal major subset," or "there exists an atomless $r$-maximal set." Thus, several new theorems on r.e. sets must be proved in order to construct the canonical realizations.

A major new source of difficulty is that the "outermost set" $A_{1}$ of $L_{1}$ and $L_{2}$ shown in Figures 2 and 3 need no longer be maximal. Thus in $\mathcal{L}^{\prime}$ for Figure 1 we may need to construct $B \in \mathcal{E}$ such that $A_{1} \subset_{\infty} B \subset_{\infty} \omega$, and such that the d.r.e. set $B-A_{1}$ joined with various d.r.e. pieces of $\mathcal{L}^{\prime}$ is or is not r.e. There is no counterpart to this in Lachlan's procedure.

Finally, the refinement theorem for $\mathrm{L}^{+}$is now more complicated since we can no longer always reduce to cases of lower characteristic, but must give a different procedure for effectively choosing a finite set of canonical realizations as potential counterexamples.

The next step in this program is to add further predicates distinguishing the $r$-maximal sets and major subsets. The new theorems proved (such as those on small or $r$-maximal major subsets) will be those required for the next canonical realizations and will not merely be random facts.

In summary, the major recent results on $\mathcal{E}$ emphasize that further progress on automorphisms or decidability of $\mathcal{E}^{*}$ will depend upon a deep knowledge of when a certain finite union of d.r.e. sets is r.e., i.e. when an element of $\mathbb{Q}^{*}$ is in $\mathcal{E}^{*}$. The definitions of $d$-simplicity and smallness and their associated results are steps in this direction. The refinement theorems give methods for arranging that such a union is or is not r.e.

\section{CHAPTER III. THE STRUCTURE OF THE R.E. DEGREES}

11. Basic facts. The study of the structural properties of degrees was begun by Kleene and Post [KIPo]. The r.e. degrees $\mathbf{R}$ have a smallest member and every pair a, $\mathbf{b} \in \mathbf{R}$ has a least upper bound, namely $\operatorname{dg}(A) \cup \operatorname{dg}(B)=\operatorname{dg}(A$ $\oplus B$ ) where $A \oplus B=\{2 x: x \in A\} \cup\{2 x+1: x \in B\}$. Unfortunately, $\mathbf{R}$ does not form a lattice because Lachlan [La5, p. 569] showed that there exist $\mathbf{a}, \mathbf{b} \in \mathbf{R}$ such that $\mathbf{a}$ and $\mathbf{b}$ have no greatest lower bound. (Indeed by Lachlan's proof the original Friedberg-Muchnik degrees have this property [So1].) Thus, the structure $(R, \leqslant, U)$ forms an upper semi-lattice with least element $0=\operatorname{dg}(\phi)$ and greatest element $0^{\prime}=\operatorname{dg}(K)$.

Little is known about the automorphisms or decidability questions for $\mathbf{R}$, and its structure theorems are less coherent than those for $\mathcal{E}$. Thus, we have organized this chapter around methods of proof, and we shall stress certain broad themes and programs as they arise.

12. The finite injury priority method. In the priority method an r.e. set $A$ is constructed by stages to meet certain requirements $\left\{R_{n}\right\}_{n<\omega}$. If $n<m$, requirement $R_{n}$ is given priority over $R_{m}$ and action taken for $R_{m}$ at some stage $s$ may be undone at a later stage $t>s$ for the sake of $R_{n}$, thereby injuring $R_{m}$ at stage $t$. The finite injury priority method invented by Friedberg and Muchnik is characterized by the fact that each requirement is injured at 
most finitely often. Let $\Phi_{e}(A)=B$ denote the eth algorithm given $A$ as "oracle" computes $B$. Now $B \leqslant_{T} A$ iff $\Phi_{e}(A)=B$ for some $e$. Let $\Phi_{e, s}(A ; x)$ denote the result if any after performing $s$ steps in the eth algorithm with oracle $A$ and input $x$. We identify sets with their characteristic functions and let $A[z]$ be the characteristic function of $A$ restricted to arguments $\leqslant z$. If $\Phi_{e, s}(A, x)$ is defined then there exist $z$ and $y$ such that $\Phi_{e, s}(A[z] ; x)=y$, and if $B[z]=A[z]$ then $\Phi_{e, s}(B ; x)=y$ also. (Thus, $\Phi_{e}$ is an effectively continuous functional from the Cantor space $2^{\omega}$ to itself.)

TheOREM 12.1 (Friedberg-MuchNik). There exist r.e. sets $A$ and $B$ such that $A \nless T B$ and $B \nless T A$.

SKETCH OF PROOF. It suffices to meet for all $e$ the requirements,

$$
R_{2 e}: \Phi_{e}(A) \neq B \text { and } R_{2 e+1}: \Phi_{e}(B) \neq A \text {. }
$$

To meet $R_{2 e}$ the strategy is to choose a "witness" $x \notin B_{s}$, and wait until $\Phi_{e, s}\left(A_{s}[z], x\right)=y$ is defined for some $s, z$, and $y$. Then enumerate $x$ in $B$ just if $y=0$, define the restraint function $r(2 e, s+1)=z$, and attempt to "restrain" all elements $v \leqslant r(2 e, s+1)$ from entering $A$ (so that $\Phi_{e}(A, x) \neq$ $B(x)$ ). If some $v \leqslant r(2 e, s+1)$ later enters $A$ (thereby probably destroying the computation above), then $R_{2 e}$ is injured and the strategy for $R_{2 e}$ begins all over on a new witness. Since $R_{e}$ is injured only by the action of $R_{j}, j<e, R_{e}$ is injured at most finitely of ten and is eventually satisfied forever, and $\lim _{s} r(e, s)<\infty$.

Friedberg and Muchnik also showed [Fr1] that for any $\mathbf{a} \in \mathbf{R}^{+}$there exists $\mathbf{b} \in \mathbf{R}^{+}, \mathbf{b}<\mathbf{a}$. Hence, there are infinitely many r.e. degrees. Using the same method Sacks proved [Sa7] that there is an r.e. sequence of r.e. sets $\left\{A_{n}\right\}_{n \in \omega}$ recursively independent in the sense that $A_{n} \nless \tau\left\{\langle x, m\rangle: x \in A_{m}\right.$ and $m \neq$ $n$ \}.

Corollary 12.2 (SACKs). Any countable partially ordered set can be embedded in $\mathbf{R}$ (as a poset).

Stronger embeddings of lattices into $\mathbf{R}$ (preserving least and greatest elements and $U$ and $\cap$ where possible) will be considered later. Sacks later extended this method to prove his splitting theorem (Theorem 6.3) which implies

CoROllary 12.3 (SACKs). Every nonzero r.e. degree is the join of a pair of incomparable low r.e. degrees.

Sacks' method has been extremely useful. For a simple illustration of this method see [S04, Theorem 1.1], where it is used to prove that for every nonrecursive r.e. set $C$ there is a simple r.e. set $A$ such that $C \Varangle_{T} A$. (To satisfy a requirement of the form $\Phi_{e}(A) \neq C$, Sacks ingeniously preserves agreement between $\Phi_{e, s}\left(A_{s} ; x\right)$ and $C_{s}(x)$ rather than disagreement as in the Friedberg-Muchnik theorem, and this preservation guarantees that if $\Phi_{e}(A)$ $=C$ then $C$ is recursive, contrary to hypothesis.)

13. The infinite injury priority method. A much more powerful method (where a requirement may be injured infinitely often) was discovered by 
Shoenfield [SF3] and independently by Sacks [Sa3] who developed it and applied it to degrees [Sa4], [Sa5], [Sa7], as did Yates [Y4], [Y6]. A detailed exposition of this method can be found in [S04] or [La14]. Perhaps the easiest example of this method is the Thickness Lemma. For $A \subseteq \omega$, and $x \in \omega$ define the "column" $A^{(x)}=\{\langle x, y\rangle:\langle x, y\rangle \in A\}$. A subset $A \subseteq B$ is a thick subset of $B$ if $A^{(x)}={ }^{*} B^{(x)}$ for all $x$, and $B$ is piecewise recursive if $B^{(x)}$ is recursive for each $x$.

Shoenfield first proved [Sf3] a weaker version of the following lemma (with $C \equiv_{T} K$ ) in order to study representability of recursive sets and functions in theories. To replace $K$ by a nonrecursive r.e. set $C$ we need to add the above Sacks strategy of preserving agreement.

TheOREM 13.1 (ThICKNESS LeMMA-ShOENFIELD). Given a nonrecursive r.e. set $C$ and a piecewise recursive set $B$ there is an r.e. thick subset $A$ of $B$ such that $C \nless T A$.

SKETCH OF PROOF. One must meet the requirements,

$$
P_{e}: B^{(e)}={ }^{*} A^{(e)} \text { and } N_{e}: C \neq \Phi_{e}(A) .
$$

A positive requirement like $P_{0}$ may infinitely often injure some negative requirement $N_{e}$ by infinitely often forcing $v \in A_{s+1}-A_{s}$ for some $v<$ $r(e, s)$, where $r(e, s)$ is the maximum of those elements used in computations of the form $\Phi_{e, s}\left(A_{s}, x\right)$ for $x<l(e, s)$, where

$$
l(e, s)=\max \left\{x:(\forall y<x)\left[C_{s}(y)=\Phi_{e, s}\left(A_{s} ; y\right)\right]\right\} .
$$

Quite surprisingly, minor modifications of the construction and proofs of the Sacks finite injury case suffice for the infinite injury case as well [S04, 82]. For $N_{e}$ define the injury set

$$
I_{e}=\left\{x:(\exists s)\left[x \in A_{s+1}-A_{s} \text { and } x<r(e, s)\right]\right\} .
$$

The reason $N_{e}$ can still be satisfied even though $I_{e}$ is no longer finite is that $I_{e}$ is recursive because $B$ is piecewise recursive. Thus, if $\Phi_{e}(A)=C$ we can still conclude that $C$ is recursive contrary to hypothesis.

The Thickness Lemma and its extensions have numerous consequences for r.e. degrees, such as the existence of an incomplete high degree, and the fact that $0^{\prime}$ is not a minimal upper bound for any infinite simultaneously r.e. sequence $d_{0}<d_{1}<\ldots$ of r.e. degrees (Sacks). (On the other hand Cooper [Cp2] has used a different proof to show that some low r.e. degrees are minimal upper bounds for such sequences, and Shore has noted that this yields an alternate proof that $R$ is not a lattice.)

Using the infinite injury method Sacks proved two of the most important theorems on r.e. degrees [Sa3], [Sa5].

THEOREM 13.2 (JUMP THEOREM-SACKs). For any degree $\mathrm{d}$ r.e. in $\mathbf{0}^{\prime}$ such that $\mathbf{0}^{\prime}<\mathrm{d}$ there exists an r.e. degree a such that $\mathbf{a}^{\prime}=\mathbf{d}$. (Furthermore, for any nonzero degree $\mathrm{c}<0^{\prime}$ we may choose a $\neq \mathrm{c}$.)

TheOREM 13.3 (Density TheOREM-SACKS). For any r.e. degrees $\mathbf{a}<\mathbf{b}$ there is an r.e. degree $\mathbf{c}$ such that $\mathbf{a}<\mathbf{c}<\mathbf{b}$. 
The Jump Theorem implies that for all $n, \mathbf{H}_{n} \subsetneq \mathbf{H}_{n+1}$ and $\mathbf{L}_{n} \subsetneq \mathbf{L}_{n+1}$. Another corollary [Sa8] or [Rg2, p. 294] is that $R \neq \bigcup_{n}\left(\mathbf{L}_{n} \cup \mathbf{H}_{n}\right)$ which was first proved by Lachlan [La2] and Martin [Ma4]. The Density Theorem is probably the most pleasing property of the r.e. degrees and distinguishes $\mathbf{R}$ from the non-r.e. degrees where minimal degrees exist.

Yates [Y4], [Y6], and later Kallibekov [Ka] used the method to classify index sets such as $\left\{e: W_{e} \equiv_{T} A\right\}$ for some r.e. set $A$, and to give an alternate proof of the Density Theorem. Numerous other results using this method were proved by Sacks [Sa7] and Robinson [Ro6] and [Ro7], some of which may be found in [So4]. For example,

THEOREM 13.4 (Jump INTERPOlATION THEOREM-RoBinson). Given r.e. degrees $\mathbf{d}<\mathbf{c}$ and a degree $\mathrm{b}$ r.e. in $\mathbf{c}$ such that $\mathrm{d}^{\prime}<\mathrm{b}$, then there exists an r.e. degree a such that $\mathbf{d}<\mathbf{a}<\mathbf{c}$ and $\mathbf{a}^{\prime}=\mathbf{b}$.

This implies both the Jump Theorem and Density Theorem and its proof combines the strategies for proving each.

14. The minimal pair method and lattice embeddings in $R$. Consider the language $L(<)$ for describing posets which has a binary symbol $<$ and whose atomic formulas are of the form $x<y$ or $x=y$. Let $L(<, U)$ denote the extended language where atomic formulas of the form $x \cup y=z$ are added, and similarly $L(<, \cup, 0,1)$ where 0 and 1 are interpreted in $R$ as the least and greatest elements 0 and $0^{\prime}$. After seeing the Density Theorem Shoenfield attempted to describe $\mathbf{R}$ algebraically by formulating a conjecture [Sf4] which implies that $\mathbf{R}$ is a dense structure as a poset analogously as the rationals are a dense structure as a linearly ordered set. Shoenfield's conjecture implies that if $\varphi\left(x_{1}, \ldots, x_{n}, y\right)$ is a quantifier free formula of $L(<, \cup, 0,1)$ and $a_{1}, \ldots, a_{n} \in \mathbf{R}$ then there exists $\mathbf{b} \in \mathbf{R}$ such that $\varphi\left(\mathbf{a}_{1}, \ldots, \mathbf{a}_{n}, \mathbf{b}\right)$ holds, unless the existence of $\mathbf{b}$ would lead to an "inconsistency." (For logicians, Shoenfield's conjecture asserts that $\mathbf{R}$ is countably saturated in $L(<, \cup, 0,1)$ with respect to quantifier free formulas.) In addition to the Density Theorem, consequences of Shoenfield's conjecture for $\mathbf{R}$ are:

If $\mathbf{a}, \mathbf{b} \in \mathbf{R}$ are incomparable then they have no greatest lower bound in $\mathbf{R}$.

Given r.e. degrees $\mathbf{0}<\mathbf{b}<\mathbf{a}$ there exists an r.e. degree $\mathbf{c}<\mathbf{a}$ such that $\mathbf{a}=\mathbf{b} \cup \mathbf{c}$.

Unfortunately both of these consequences are false (Theorem 14.1 and \$15) but as Shoenfield anticipated [Sf4, p. 363] they have led to the development of two important new areas and techniques of proof. We say that $\mathbf{a}, \mathbf{b} \in R^{+}$ form a minimal pair if $\mathbf{a} \cap \mathbf{b}=\mathbf{0}$. Minimal pairs were constructed by Lachlan [La5] and independently by Yates [Y3].

Theorem 14.1 (Minimal Pair Theorem-Lachlan-Yates). There exist nonrecursive r.e. degrees $\mathbf{a}$ and $\mathbf{b}$ such that $\mathbf{a} \cap \mathbf{b}=0$.

SKETCH OF PROOF. It suffices to construct r.e. sets $A$ and $B$ satisfying for all $e$ the requirements 


$$
\begin{aligned}
& N_{e}: \Phi_{e}(A)=\Phi_{e}(B)=f \Rightarrow f \text { is a recursive function, and } \\
& P_{e}^{A}: W_{e} \cap A \neq \varnothing \text { if } W_{e} \text { is infinite, }
\end{aligned}
$$

and similarly $P_{e}^{B}$ with $B$ in place of $A$. As in $\S 12$ the positive requirements $P_{e}$ are finitary since each contributes at most one element to $A$. If $W_{e, s} \cap A_{s}=\varnothing$ certain elements $x \in W_{e, s}$ may be appointed as followers of $P_{e}^{A^{e}}$ since their later enumeration in $A$ would satisfy $P_{e}^{A}$.

The strategy for meeting $N_{e}$ and still allowing followers $u$ of $P_{i}^{A}$ to enter $A$ and $v$ of $P_{i}^{B}$ to enter $B$ is the following. First define

$$
l(e, s)=\max \left\{x:(\forall y<x)\left[\Phi_{e, s}\left(A_{s} ; y\right)=\Phi_{e, s}\left(B_{s} ; y\right)\right]\right\} .
$$

Now enumerate $u$ in $A$ but restrain from $B$ all elements $z \leqslant r(e, s+1)$, where $r(e, s+1)$ is defined to be the maximum element used in a computation $\Phi_{e, s}\left(B_{s} ; x\right)$ for some $x \leqslant l(e, s)$. (Enumeration of $x$ in $A_{s+1}-$ $A_{s}$ may destroy some computation $\Phi_{e, s}\left(A_{s} ; x\right)$ and cause $l(e, s+1)<$ $l(e, s)$.) Now wait for a stage $t>s$ such that $l(e, t) \geqslant l(e, s)$. If $t$ never exists then $\Phi_{e}(A) \neq \Phi_{e}(B)$ so $N_{e}$ is automatically met and we choose a new follower $v^{\prime}>r(e, s)$ for $P_{i}^{B}$. If $t$ exists then set $r(e, t)=0$, allow $v$ to enter $P_{i}^{B}$ and at stage $t+1$ let $r(e, t+1)$ be the maximum element used in $\Phi_{e, t}\left(A_{t}, x\right)$, for any $x \leqslant l(e, t)$, and attempt to restrain all $v \leqslant r(e, t+1)$ from entering $A$ in order to preserve the $A$-computations until $l\left(e, t^{\prime}\right) \geqslant l(e, t)$ for some $t^{\prime}>t$. Now for any $p<l(e, s)$, if $\Phi_{e, s}\left(A_{s}, p\right)=q$ then for all $t \geqslant s$, either $\Phi_{e, t}\left(A_{t}, p\right)=q$ or $\Phi_{e, t}\left(B_{t} ; p\right)=q$ so $f(p)=q$ may be recursively computed.

An elegant exposition of this argument using the model of a "pinball machine" to give a clear picture of the construction may be found in Lerman [Le6]. A follower $u$ of $P_{i}^{A}$ enters the machine like a "ball" and falls downwards past all "gates" $G_{e}, e \leqslant i$, in decreasing order of index. Gate $G_{e}$ corresponds to requirement $N_{e}$, and $G_{e}$ releases an element $x$ at stage $s$ to pass to $G_{e-1}$ roughly when $x \geqslant r(e, s)$ as defined above. The crucial point is that by the above procedure at most finitely many elements are permanently restrained at any gate $G_{e}$, i.e., $\lim \inf _{s} r(e, s)<\infty$. Lachlan [La14] gives another exposition of this proof and a proof of the density theorem as effective analogues of the Baire category theorem in topology.

We say $\mathbf{a} \in \mathbf{R}$ bounds a minimal pair if there exists a minimal pair $\mathbf{b}, \mathbf{c}$ such that $\mathbf{b} \leqslant \mathbf{a}$ and $\mathbf{c} \leqslant \mathbf{a}$.

THEOREM 14.2 (COOPER). Every degree $\mathbf{a} \in \mathbf{H}_{1}$ bounds a minimal pair.

(Cooper's proof [Cp6] combines the minimal pair construction with the Martin permitting method below a high degree (Theorem 3.6) but the permitting is associated with the negative requirements $N_{e}$, rather than the positive requirements $\boldsymbol{P}_{e}$ as in Martin, so Cooper can show that only finitely many elements are permanently held at any gate $G_{e}$.)

Lachlan [La19] has proved that there exist $\mathbf{a}, \mathbf{b} \in \mathbf{R}^{+}$such that a bounds no minimal pair, but every nonrecursive r.e. $\mathbf{c}<\mathbf{b}$ bounds a minimal pair. The exact classification of degrees bounding minimal pairs remains open.

Returning to the embedding question raised after Corollary 12.2 let us consider the language $L^{\prime}=L(\leqslant, \cup, \cap, 0)$. (The formulas of $L$ are as 
expressive as those of $L^{\prime}$, but the existential formulas of $L^{\prime}$ are much more powerful. A formula is existential if it is of the form $\left(\exists y_{1}\right) \ldots$ $\left(\exists y_{m}\right) \varphi\left(x_{1}, \ldots, x_{n}, y_{1}, \ldots, y_{m}\right)$ where $\varphi$ has no quantifiers.) For posets $X$ and $Y$ with least element an $L^{\prime}$-embedding $f$ is a 1: 1 mapping from $X$ into $Y$ which preserves $0, \cup, \cap$, and $\leqslant$. A major open question on embeddings has been posed by Lachlan, Robinson [Ro6, p. 313], and by Shoenfield [Sf6, p. 976].

ConjeCture 14.3 (Embedding Conjecture). Every finite lattice has an $L^{\prime}$ embedding in $\mathbf{R}$.

The Embedding Conjecture (E.C.) if true would give a decision procedure for existential sentences of $L^{\prime}$ true in $R$, and would salvage some of the algebraic uniformity of $\mathbf{R}$ implied by Shoenfield's conjecture. The E.C. has been proved by Thomason [Th] and independently by Lerman (unpublished) for distributive lattices, and by Lachlan [La3] for the two five-element nondistributive lattices. The principal method of proof is the minimal pair construction. Although greater difficulties arise in the nondistributive case these can be reasonably handled with the pinball machine model.

In attempting to prove E.C. for the general case the major difficulty is preserving a meet when it lies above a nondistributive lattice. In particular, if one could prove E.C. for the following lattice shown in Figure 4, one could probably do the general case.

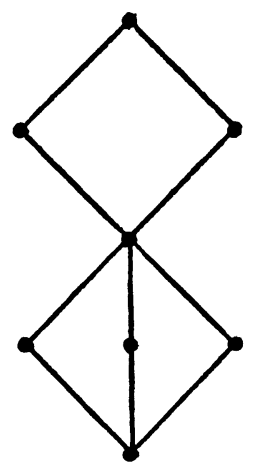

FIGURE 4

Lachlan [La5, Theorem 5] has shown that E.C. is false in the language $L^{\prime \prime}=L(\leqslant, \cup, \cap, 0,1)$.

Theorem 14.4 (Non-Diamond Theorem-Lachlan). There are no incomparable r.e. degrees $\mathbf{a}, \mathbf{b}$ such that $\mathbf{a} \cup \mathbf{b}=\mathbf{0}^{\prime}$ and $\mathbf{a} \cap \mathbf{b}=\mathbf{0}$.

This result is surprising and its proof is entirely new and clever. (Note that the Non-Diamond Theorem and Corollary 12.3 imply that $\mathbf{R}$ does not satisfy the Reduction Principle since if incomparable r.e. degrees $\mathbf{a}$ and $\mathbf{b}$ satisfy $a \cup b=0^{\prime}$, no $a_{1} \leqslant a$ and $b_{1} \leqslant b$ can satisfy $a_{1} \cup b_{1}=0^{\prime}$ and $a \cap b=0$.) Lachlan [La5, p. 568] and later Robinson [Ro6, p. 313] then ask for the following generalization.

CONJECTURE 14.5. If $\mathbf{a}$ and $\mathbf{b}$ are incomparable r.e. degrees satisfying $\mathbf{a} \cup \mathbf{b}=\mathbf{0}^{\prime}$ then $\mathbf{a}$ and $\mathbf{b}$ have no greatest lower bound in $\mathbf{R}$. 
Positive solution of Conjectures 14.3 and 14.5 would assist in finding a decision procedure for certain classes of sentences true of $\mathbf{R}$. In particular, they assert that the finite lattices which can be $L^{\prime \prime}$-embedded into $\mathbf{R}$ are those such that 1 is not the join of elements other than 1 .

Let $\mathbf{M}$ consist of $\mathbf{O}$ and those degrees which form half of a minimal pair. Jockusch has extended Lachlan's Theorem 14.4 by showing that no finite join of degrees in $\mathrm{M}$ can be $\mathbf{0}^{\prime}$. It follows from Corollary 12.3 that there is a low degree $\mathbf{a} \in \mathbf{R}$ such that $\mathbf{a} \nless \mathbf{b} \cup \mathbf{c}$ for any $\mathbf{b}, \mathbf{c} \in M$. Thus, as Harrington first observed, $0^{\prime}$ may be replaced by a low r.e. degree in Theorem 14.4.

By analogy with the simple sets define the simple r.e. degrees $\mathbf{S}=\mathbf{R}-\mathbf{M}$. (We include $\mathbf{0} \in \mathbf{M}$ so that $\mathbf{M}$ is closed downwards under $<$, and similarly we include $0^{\prime} \in \mathbf{S}$.) Since the main obstructions to Shoenfield's conjecture in Theorems 14.1 and 14.4 arise from nonsimple degrees, Jockusch has conjectured that the original Shoenfield conjecture holds for the simple degrees together with $\mathbf{0}$.

Other results about minimal pairs, simple degrees and nonbranching degrees may be found in Yates [Y3] and Lachlan [La5]. (An r.e. degree $a$ is branching if there are r.e. degrees $\mathbf{b}, \mathbf{c}$ both $>\mathbf{a}$ and such that $\mathbf{a}=\mathbf{b} \cap \mathbf{c}$, and $a$ is nonbranching otherwise. The minimal pair theorem asserts that $\mathbf{0}$ is branching, although Lachlan proves [La5] that below every $\mathbf{a} \in \mathbf{R}^{+}$there is a nonbranching $\mathbf{b} \in \mathbf{R}^{+}$.)

15. Cupping and splitting r.e. degrees. $A$ degree $a \in R$ has the anticupping (a.c.) property if there exists a nonrecursive r.e. $b<$ a such that for no r.e. $c<\mathbf{a}$ does $\mathbf{a}=\mathbf{b} \cup \mathbf{c}$. Consequence (14.2) of Shoenfield's conjecture asserts that no $a \in R$ has the a.c. property. Lachlan disproved (14.2) in [La4]. Furthermore, every $\mathbf{a} \in \mathbf{R}^{+}$has a predecessor $\mathbf{b} \in \mathbf{L}_{2} \cap \mathbf{R}^{+}$with the a.c. property [LdSs]. Yates, Cooper [Cp7] and Harrington proved that $\mathbf{O}^{\prime}$ has the a.c. property and Harrington proved that all degrees $a \in \mathbf{H}_{1}$ have it.

(A new method of proof is required for this result since the restraint function $r(e, s)$ for the negative requirements may have the property that $\lim \inf _{s} r(e, s)=\infty$, unlike the arguments in $\$ 13$ and $\S 14$ where lim inf $r(e, s)<\infty$. The solution is to modify the pinball machine model and "spread out" the restraint associated with negative requirement $N_{e}$ over all gates $G_{i}, i \geqslant e$ (not merely at $G_{e}$ as before) so it will still be true that only finitely many elements are permanently restrained at gate $G_{e}$.)

Using yet another new method of Lachlan [La6] Harrington proved that not every $a \in \mathbf{R}^{+}$has the a.c. property, but the exact classification of such degrees remains open. Also unclassified is the set of degrees $\mathbf{C}$ consisting of

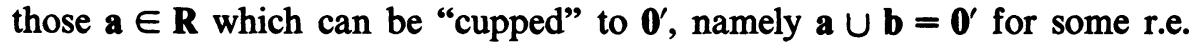
$\mathbf{b}<\mathbf{0}^{\prime}$. (Note that $\mathbf{C}$ is exactly dual to $\mathbf{M}$ with $\cup$ and $\boldsymbol{O}^{\prime}$ in place of $\cap$ and $\mathbf{0}$.) Harrington proved that there exists a high r.e. degree $\mathbf{a}<\mathbf{0}^{\prime}$ such that $\mathbf{a} \notin \mathbf{C}$. He also proved that $\mathbf{C} \cap \mathbf{M} \neq \varnothing$ and $\mathbf{C} \cup \mathbf{M}=\mathbf{R}$.

Returning to splitting theorems, Robinson used the recursion theorem [Ro6, Corollary 9] to generalize the Sacks Splitting Theorem, Corollary 12.3 (which constitutes the case $\mathbf{c}=\mathbf{0}$ below).

Theorem 15.1 (RoBInSon). Given r.e. degrees $\mathbf{c}<$ a such that $\mathbf{c} \in \mathbf{L}_{1}$ there 
exist incomparable r.e. degrees $\mathbf{b}_{0}$, $\mathbf{b}_{1}$ such that $\mathbf{c}<\mathbf{b}_{i}<\mathbf{a}$ for $i<2$, and $\mathbf{a}=\mathbf{b}_{\mathbf{0}} \cup \mathbf{b}_{\mathbf{1}}$.

Like Theorem 4.1 this theorem emphasizes the resemblance of low r.e. sets and degrees to recursive ones. Lachlan has proved [La16] that Theorem 15.1 fails in general for r.e. degrees $\mathbf{c}<\mathbf{a}$. Lachlan's proof is complicated. However, the method is new, ingenious, and very powerful. It has already been applied to obtain most of the results above by Harrington on $\mathbf{C ,} \mathbf{M}$ and the a.c. property, and it promises further application.

16. Automorphisms and decidability of $\mathbf{R}$. There are no known nontrivial automorphisms of the poset $(\mathbf{R}, \leqslant)$, and the methods of $\$ 9$ for generating automorphisms of $\mathcal{E}$ apparently do not apply to $\mathbf{R}$. Thus, attention has turned to automorphism bases in the sense of \$9. Lerman [Le7] has shown that for any degree $d \geqslant 0^{\prime}$ and r.e. in $0^{\prime}$, the class $B_{d}=\left\{a: a \in R\right.$ and $\left.a^{\prime}=d\right\}$ forms an automorphism basis for $\mathbf{R}$. In particular, each class of the form $\mathbf{L}_{n}$, $\mathbf{H}_{n}, \mathbf{L}_{n+1}-\mathbf{L}_{n}$, or $\mathbf{H}_{n+1}-\mathbf{H}_{n}$ for $n \geqslant 1$ forms an automorphism basis. In addition there are other automorphism bases. If enough such bases are found it may be possible to prove that there are no nontrivial automorphisms.

Very little is known about the decidability of the elementary theory of $\mathbf{R}$. Lachlan proved undecidability of the elementary theory of degrees by showing that every finite distributive lattice can be embedded as an initial segment of the degrees. This method fails for $\mathbf{R}$ by the Density Theorem.

Recently Shore has produced a decidable subclass of the $\vec{\forall} \vec{\exists}$-sentences of $L(<, 0,1)$ true in $\mathbf{R}$. He has decided all sentences $S$ of the form $(\forall \vec{x})(\exists \vec{y})[D(\vec{x}) \Rightarrow P(\vec{x}, \vec{y})]$ where $D(\vec{x})$ is an atomic diagram for the $x$ 's and $P(\vec{x}, \vec{y})$ is a complete atomic diagram for the $x$ 's and $y$ 's. (The difference between Shore's result for $\mathbf{R}$ and Lachlan's for $\mathcal{E}^{*}$ is that Lachlan allows $P(x, y)$ to be a finite disjunction $\bigvee\left\{Q_{i}(\vec{x}, \vec{y}): i \leqslant k\right\}$ of complete diagrams $Q_{i}(\vec{x}, \vec{y})$, and hence Shore does not decide all $\vec{\forall} \vec{\exists}$-sentences.)

Shore accomplishes this by using minimal pair type results of $\$ 14$ (specifically embeddings of finite distributive lattices) to construct the equivalent of Lachlan's canonical realizations of $\$ 10$ to provide necessary conditions for $S$ to be true. He then uses an infinite injury type argument as in $\$ 13$ to produce the equivalent of Lachlan's refinement theorem.

Future results on r.e. degrees should be aimed at extending this procedure, first for disjunctions of complete diagrams (and hence for all $\vec{\forall} \vec{\exists}$-sentences of $L(<, 0,1)$ true in $\mathbf{R})$, and then for $\vec{\forall} \vec{\exists}$-sentences in stronger languages such as $L(\leqslant, \cup, \cap, 0,1)$. Positive solution of the conjectures in $\S 14$ should be very helpful in both, particularly the former.

ADDED JULY 10, 1978. Conjecture 14.4 has been refuted by Shoenfield and Soare and independently by Lachlan. Indeed Lachlan has shown that for any nonzero r.e. degree $\mathbf{a}$ there are incomparable r.e. degrees $\mathbf{b}_{0}$, $\mathbf{b}_{1}$ having an infimum such that $\mathbf{a}=\mathbf{b}_{0} \cup \mathbf{b}_{1}$.

\section{BIBLIOGRAPHY}

[A1] D. A. Alton, Uniformities in recursively enumerable sets, Doctoral Dissertation, Cornell Univ., Ithaca, N. Y., 1970. 
[A2] Recursively enumerable sets which are uniform for finite extensions, J. Symbolic Logic 36 (1971), 271-287.

[BeSo] V. L. Bennison and R. I. Soare, Some lowness properties and computational complexity sequences, Theor. Comput. Sci. (to appear).

[Bi1] G. Birkhoff, On the combination of subalgebras, Proc. Cambridge Philos. Soc. 29 (1933), 441-464.

[Bi2] _ Lattice theory, 3rd ed., Amer. Math. Soc. Colloq. Publ., vol. 25, Amer. Math. Soc., Providence, R I., 1967.

[BIMq] M. Blum and I. Marques, On complexity properties of recursively enumerable sets, J. Symbolic Logic 38 (1973), 579-593.

[Bo1] W. W. Boone, Certain simple unsolvable problems of group theory, Indag. Math. 16 (1954), 231-237, 492-497; ibid. 17 (1955), 252-256, 571-577; ibid. 19 (1957), 22-27, 227-232.

[B02] _ The word problem, Ann. of Math. (2) 70 (1959), 207-265.

[B03] _ـ Word problems and recursively enumerable degrees of unsolvability, $A$ sequel on finitely presented groups, Ann. of Math. (2) 84 (1966), 49-84.

[CoJ] P. Cohen and C. G. Jockusch, Jr., A lattice property of Post's simple set, Illinois J. Math. 19 (1975), $450-453$.

[Ch] A. Church, An unsolvable problem of elementary number theory, Amer. J. Math. 58 (1936), 345-363.

[Cp1] S. B. Cooper, Sets recursively enumerable in high degrees, Notices Amer. Math. Soc. 19 (1972), A-20.

[Cp2] __, Minimal upper bounds for sequences of recursively enumerable degrees, J. London Math. Soc. 5 (1972), 445-450.

[Cp3] __ Degrees of unsolvability complementary between recursively enumerable degrees I, Ann. Math. Logic 4 (1972), 31-73.

[Cp4] _ Jump equivalence of the $\Delta_{2}^{0}$ hyperhyperimmune sets, J. Symbolic Logic 37 (1972) 598-600.

[Cp5] __ An annotated bibliography for the structure of the degrees below $\sigma^{\gamma}$ with special reference to that of the recursively enumerable degrees, Recursive Function Theory Newsletter 5 (1974), 1-15.

[Cp6] __, Minimal pairs and high recursively enumerable degrees, J. Symbolic Logic 39 (1974), 655-660.

[Cp7] , On a theorem of C. E. M. Yates (preprint), 1974.

[Da1] M. Davis, Computability and unsolvability, McGraw-Hill, New York, 1958.

[Da2] $\longrightarrow$ (Ed.), The undecidable. Basic papers on undecidable propositions, unsolvable problems and computable functions, Raven Press, Hewlitt, N. Y., 1965.

[Da3] __ Hilbert's tenth problem is unsolvable, Amer. Math. Monthly 80 (1973), 233-269.

[De] A. N. Degtev, Hypersimple sets with retraceable complements, Algebra i Logika 10 (1971), 235-246.

[Dk1] J. Dekker, Two notes on recursively enumerable sets, Proc. Amer. Math. Soc. 4 (1953), 495-501.

[Dk2] __ A theorem on hypersimple sets, Proc. Amer. Math. Soc. 5 (1954), 791-796.

[Dk3] __ Productive sets, Trans. Amer. Math. Soc. 78 (1955), 129-149.

[DkMy1] J. C. E. Dekker and J. Myhill, Some theorems on classes of recursively enumerable sets, Trans. Amer. Math. Soc. 89 (1958), 25-29.

[DkMy2] _ Retraceable sets, Canad. J. Math. 10 (1958), 357-373.

[E1] Y. L. Ershov, Decidability of the elementary theory of relatively complemented distributive lattices and of the theory of filters, Algebra i Logika 3 (1964), 17-38. (Russian)

[E2] __ A hierarchy of sets, Parts I, II, III, Algebra and Logic 7 (1968), 25-43, 212-232; ibid. 9 (1970), 20-31.

[Fe] S. Feferman, Degrees of unsolvability associated with classes of formalized theories, J. Symbolic Logic 22 (1957) 161-175.

[Fn] L. Feiner, Orderings and Boolean algebras not isomorphic to recursive ones, Doctoral Dissertation, M.I.T., Cambridge, Mass., 1967.

[Fr1] R. M. Friedberg, The fine structure of degrees of unsolvability of recursively enumerable sets, Seminars of Cornell Institute for Symbolic Logic, 1957, pp. 404-406.

[Fr2] _ Two recursively enumerable sets of incomparable degrees of unsolvability, Proc. Nat. Acad. Sci. U.S.A. 43 (1957), 236-238. MR 18 \#867. 
[Fr3] (1957), 159-160.

A criterion for completeness of degrees of unsolvability, J. Symbolic Logic 22

[Fr4] __, Three theorems on recursive enumeration: I. Decomposition, II. Maximal set, III. Enumeration without duplication, J. Symbolic Logic 23 (1958), 309-316. MR 22 \# 13.

[FrRg] R. M. Friedberg and H. Rogers, Jr., Reducibility and completeness for sets of integers, $\mathbf{Z}$. Math. Logik Grundlagen Math. 5 (1959), 117-125.

[GiMr] J. Gill and P. Morris, On subcreative sets and S-reducibility, J. Symbolic Logic 39 (1974), 669-677.

[Gö1] K. Gödel, Über formal unentscheidbare Sätze der Principia Mathematica und verwandter Systeme. I, Monatsh. Math. Phys. 38 (1931), 173-198.

[Gö2] _ On undecidable propositions of formal mathematical systems, Notes by S. C. Kleene and Barkley Rosser on lectures at the Institute for Advanced Study, Princeton, N. J., 1934. Reprinted in Davis, [Da2].

[Ha] W. Hanf, Model theoretic methods in the study of elementary logic, Sympos. Theory of Models (Berkeley), North-Holland, Amsterdam, 1965, pp. 132-145.

[Hy1] L. Hay, The class of recursively enumerable subsets of a recursively enumerable set, Pacific J. Math. 46 (1973), 167-183.

[Hy2] _ The halting problem relativized to complements, Proc. Amer. Math. Soc. 41 (1973), 583-587.

[J1] C. G. Jockusch, Jr., The degrees of hyperhyperimmune sets, J. Symbolic Logic 34 (1969), 489-493.

[J2] __, Relationships between reducibilities, Trans. Amer. Math. Soc. 142 (1969), 229-237.

[J3] _ Degrees in which the recursive sets are uniformly recursive, Canad. J. Math. 24 (1972), 1092-1099.

[J4] __ Review of Lerman [Le3], MR 45 \#3200.

[J5] $\longrightarrow \mathrm{II}_{1}^{0}$ classes and Boolean combinations of recursively enumerable sets, J. Symbolic Logic 39 (1974), 95-96.

[JSo1] C. G. Jockusch, Jr. and R. I. Soare, A minimal pair of $\Pi_{1}^{0}$ classes, J. Symbolic Logic 36 (1971), 66-78.

[JSo2] __ Degrees of members of $\Pi_{1}^{0}$ classes, Pacific J. Math. 40 (1972), 605-616.

[JS03] _—, $\Pi_{1}^{0}$ classes and degrees of theories, Trans. Amer. Math. Soc. 173 (1972), 33-56.

[JS04] __ Post's problem and his hypersimple set, J. Symbolic Logic 38 (1973), 446-452.

[Ka] S. Kallibekov, Index sets of degrees of unsolvability, Algebra i Logika 10 (1971), 316-326. (Russian)

[Ke] C. F. Kent, Constructive analogues of the group of permutations of the natural numbers, Trans. Amer. Math. Soc. 104 (1962), 347-362.

[KI1] S. C. Kleene, $A$ theory of positive integers in formal logic, Amer. J. Math. 57 (1935), 153-173, 219-244.

[K12] __, General recursive functions of natural numbers, Math. Ann. 112 (1936), 727-742.

[K13] __ Introduction to metamathematics, Van Nostrand, New York, 1952. MR 14 \# 525.

[KIPo] S. C. Kleene and E. L. Post, The upper semi-lattice of degrees of recursive unsolvability, Ann. of Math. (2) 59 (1954), 379-407. MR 15 \#772.

[Ku] A. V. Kuznecov, On primitive recursive functions of large oscillation, Dokl. Akad. Nauk SSSR, 71 (1950), 233-236. (Russian)

[La1] A. H. Lachlan, Some notions of reducibility and productiveness, Z. Math. Logik Grundlagen Math. 11 (1965), 17-44.

[La2] __, On a problem of G. E. Sacks, Proc. Amer. Math. Soc. 16 (1965), 972-979.

[La3] __, A note on universal sets, J. Symbolic Logic 31 (1966), 573-574.

[La4] _ The impossibility of finding relative complements for recursively enumerable degrees, J. Symbolic Logic 31 (1966), 434-454. MR 34 \#5673.

[La5] _ Lower bounds for pairs of r.e. degrees, Proc. London Math. Soc. 16 (1966), 537-569. MR 34 \#4126.

[La6] _ The priority method. I, Z. Math. Logik Grundlagen Math. 13 (1967), 1-10.

[La7] _ Complete recursively enumerable sets, Proc. Amer. Math. Soc. 19 (1968), 99-102.

[L88] __, On the lattice of recursively enumerable sets, Trans. Amer. Math. Soc. 130 (1968), 1-37. MR 37 \#2594.

[La9] _ The elementary theory of recursively enumerable sets, Duke Math. J. 35 (1968), 123-146. MR 37 \#2593. 
[La10] _ Distributive initial segments of the degrees of unsolvability, Z. Math. Logik Grundlagen Math. 14 (1968), 457-472. MR 38 \#5620.

[La11] _ Degrees of recursively enumerable sets which have no maximal superset, $\mathrm{J}$. Symbolic Logic 33 (1968), 431-443. MR 38 \#4314.

[La12] _ On some games which are relevant to the theory of recursively enumerable sets, Ann. of Math (2) 91 (1970), 291-310. MR 44 \# 1652.

[La13] __ Embedding nondistributive lattices in the recursively enumerable degrees, Conf. Mathematical Logic, London 1970, Lecture Notes in Math., no. 255, Springer-Verlag, Berlin and New York, 1972, pp. 149-177.

[La14] _ The priority method for the construction of recursively enumerable sets, Proc. Cambridge Summer School in Logic, 1971, Lecture Notes in Math., no. 337, Springer-Verlag, Berlin and New York, 1973.

[La15] __, Recursively enumerable degrees, Handwritten notes from lectures in Warsaw, April, 1973.

[La16] A recursively enumerable degree which will not split over all lesser ones, Ann. Math. Logic 9 (1975), 307-365.

[La17] __, Uniform enumeration operations, J. Symbolic Logic 40 (1975), 401-409.

[La18] _ wtt-complete sets are not necessarily tt-complete, Proc. Amer. Math. Soc. 48 (1975), 429-434.

[La19] A. H. Lachlan, Bounding minimal pairs (to appear).

[Ld1] R. E. Ladner, A completely mitotic nonrecursive r.e. degree, Trans. Amer. Math. Soc. 184 (1973), 479-507.

[Ld2] ___ Mitotic recursively enumerable sets, J. Symbolic Logic 38 (1973), 199-211.

[LdSs] R. E. Ladner and L. P. Sasso, The weak truth table degrees of recursively enumerable sets, Ann. Math. Logic 4 (1975), 429-448.

[Le1] M. Lerman, Recursive functions modulo co-r-maximal sets, Trans. Amer. Math. Soc. 148 (1970), 429-444.

[Le2] _., Turing degrees and many-one degrees of maximal sets, J. Symbolic Logic 35 (1970), 29-40.

[Le3] _ Some theorems on r-maximal sets and major subsets of recursively enumerable sets, J. Symbolic Logic 36 (1971), 193-215. MR 45 \#3200.

[Le4] _ Congruence relations, filters, ideals, and definability in lattices of $\alpha$-recursively enumerable sets, J. Symbolic Logic 41 (1976), 405-418.

[Le5] __ Admissible ordinals and priority arguments, Proc. Cambridge Summer School in Logic, 1971, Lecture Notes in Math., no. 337, Springer-Verlag, Berlin and New York, 1973. MR 52 \# 59.

[Le6] _ Lattices of a-recursively enumerable sets, Oslo Conf. Generalized Recursion Theory, June, 1976 (to appear).

[Le7] _ On elementary theories of some lattices of a-recursively enumerable sets (to appear).

[Le8] _ Automorphism bases for the semilattice of recursively enumerable degrees, Notices Amer. Math. Soc. 24 (1977), A-251. Abstract \#77T-E10

[LeSo1] M. Lerman and R. I. Soare, $A$ decidable fragment of the elementary theory of the lattice of recursively enumerable sets, (to appear).

[LeS02] __, d-simple sets, small sets, and degree classes (to appear).

[LeShSo] M. Lerman, R. A. Shore and R. I. Soare, r-maximal major subsets, Israel. J. Math. (to appear).

[Mal] D. A. Martin, A theorem on hyperhypersimple sets, J. Symbolic Logic 28 (1963), 273-278.

[Ma2] _ Completeness, the recursion theorem, and effectively simple sets, Proc. Amer. Math. Soc. 17 (1966), 838-842.

[Ma3] _ Classes of recursively enumerable sets and degrees of unsolvability, Z. Math. Logik Grundlagen Math. 12 (1966), 295-310. MR 37 \#68.

[Ma4] __, On a question of G. E. Sacks, J. Symbolic Logic 31 (1966), 66-69.

[Ma5] _ The priority method of Sacks, mimeographed notes, 1966.

[MaMi] D. A. Martin and W. Miller, The degrees of hyperimmune sets, Z. Math. Logik Grundlagen Math. 14 (1968), 159-166.

[MaPr] D. A. Martin and M. B. Pour-El, Axiomatizable theories with few axiomatizable extensions, J. Symbolic Logic 35 (1970), 205-209. 
[Mt1] Y. Matiyasevič, Diophantine representation of enumerable predicates, Izv. Akad. Nauk. SSSR Ser. Math. 35 (1971), 3-30. (Russian)

[Mt2] , Enumerable sets are diophantine, Dokl. Akad. Nauk SSSR 191 (1970), 279-282. (Russian)

[Mc] T. G. McLaughlin, On a class of complete simple sets, Canad. Math. Bull. 8 (1965), 33-37.

[Md] Y. T. Medvedev, On nonisomorphic recursively enumerable sets, Dokl. Akad. Nauk SSSR 102 (1955), 211-214. (Russian)

[MkNe] G. Metakides and A. Nerode, Recursively enumerable vector spaces, Ann. Math. Logic, 1978 (to appear).

[Mr] C. F. Miller, On group-theoretic decision problems and their classification, Ann. of Math. Studies, no. 68, Princeton Univ. Press, Princeton, N. J., 1971.

[MoSo] M. D. Morley and R. I. Soare, Boolean algebras, splitting theorems and $\Delta_{2}^{0}$ sets, Fund. Math. 90 (1975), 45-52.

[Mu] A. A. Muchnik, On the unsolvability of the problem of reducibility in the theory of algorithms, Dokl. Akad. Nauk SSSR 108 (1956), 194-197. (Russian).

[My1] J. Myhill, Creative sets, Z. Math. Logik Grundlagen Math. 1 (1955), 97-108.

[My2] —_, The lattice of recursively enumerable sets, J. Symbolic Logic 21 (1956), 220 (abstract).

[No] P. S. Novikov, On the algorithmic unsolvability of the word problem in groups, Trudy Mat. Inst. Steklov no. 44 Izdat Akad. Nauk SSSR, Moscow, (1955). (Russian)

[01] J. C. Owings, Jr., Recursion, metarecursion and inclusion, J. Symbolic Logic 32 (1967), 173-178.

[O2] __, Review of Lachlan [La8], [La9] and Robinson [Ro3], [Ro4], J. Symbolic Logic 35 (1970), 153-155.

[Po] E. L. Post, Recursively enumerable sets of positive integers and their decision problems, Bull. Amer. Math. Soc. 50 (1944), 284-316. MR 6 \#29.

[Ri1] H. G. Rice, Classes of recursively enumerable sets and their decision problems, Trans. Amer. Math. Soc. 74 (1953), 358-366.

[R12] , On completely recursively enumerable classes and their key arrays, J. Symbolic Logic 21 (1956), 304-308.

[Ro1] R. W. Robinson, The inclusion lattice and degrees of unsolvability of the recursively enumerable sets, Doctoral Dissertation, Cornell Univ., Ithaca, N. Y., 1966.

[Ro2] __ Recursively enumerable sets not contained in any maximal set, Notices Amer. Math. Soc. 13 (1966), 325. Abstract \#632-4.

[Ro3] _ Two theorems on hyperhypersimple sets, Trans. Amer. Math. Soc. 128 (1967), 531-538. MR 35 \#6549.

[Ro4] __ Simplicity of recursively enumerable sets, J. Symbolic Logic 32 (1967), 162-172. MR 36 \# 1323.

[Ro5] , A dichotomy of the recursively enumerable sets, Z. Math. Logik Grundlagen Math. 14 (1968), 339-356. MR 38 \#5623.

[Ro6] Interpolation and embedding in the recursively enumerable degrees, Ann. of Math. (2) 93 (1971), 285-314.

[Ro7] __ Jump restricted interpolation in the r.e. degrees, Ann. of Math. (2) 93 (1971), 586-596.

[Rg1] H. Rogers, Jr., Computing degrees of unsolvability, Math. Ann. 138 (1959), 125-140.

[Rg2] , Theory of recursive functions and effective computability, McGraw-Hill, New York, 1967. MR 37 \#61.

[Rg3] _ Some problems of definability in recursive function theory, Sets, Models and Recursion Theory, John Crossley, ed., North-Holland, Amsterdam, 1967, pp. 183-201.

[Rs] B. Rosser, Extensions of some theorems of Gödel and Church, J. Symbolic Logic 1 (1936), 87-91.

[Sa1] G. E. Sacks, A minimal degree less than 0', Bull. Amer. Math. Soc. 67 (1961), 416-419.

[Sa2] __, On the degrees less than 0 , Ann of Math. (2) 77 (1963), 211-231.

[Sa3] _ Recursive enumerability and the jump operator, Trans. Amer. Math. Soc. 108 (1963), 223-239. MR 27 \#5681.

[Sa4] __, A maximal set which is not complete, Michigan Math. J. 11 (1964), 193-205. MR 29 \#3368. 
[Sa5] , The recursively enumerable degrees are dense, Ann. of Math. (2) 80 (1964),

300-312. MR 29 \#3367.

[Sa6] A simple set which is not effectively simple, Proc. Amer. Math. Soc. 15 (1964),

[Sa7] , Degrees of unsolvability, rev. ed., Ann. of Math. Studies, no. 55, Princeton Univ. Press, Princeton, N. J., 1966.

[Sa8] —_, On a theorem of Lachlan and Martin, Proc. Amer. Math. Soc. 18 (1967), 140-141.

[Sa9] $\longrightarrow R E$ sets higher up, Logic, Foundations of Mathematics and Computability Theory, 173-194, D. Reidel, Dordrecht-Holland, 1977.

[Ss] L. P. Sasso, Deficiency sets and bounded information reducibilities, Trans. Amer. Math. Soc. 200 (1974), 267-290.

[Sf1] J. R. Shoenfield, Quasicreative sets, Proc. Amer. Math. Soc. 8 (1957), 964-967.

[Sf2] __ On degrees of unsolvability, Ann. of Math. (2) 69 (1959), 644-653.

[Sf3] _ Undecidable and creative theories, Fund. Math. 49 (1961), 171-179.

[Sf4] - Application of model theory to degrees of unsolvability, Sympos. Theory of Models, North-Holland, Amsterdam, 1965, pp. 359-363. MR 34 \#53.

[Sf5] ___ Degrees of unsolvability, North-Holland, Amsterdam, 1971. MR 49 \#4768.

[Sf6] , The decision problem for recursively enumerable degrees, Bull. Amer. Math. Soc. 81 (1975), 973-977.

[S17] __ Degrees of classes of r.e. sets, J. Symbolic Logic 41 (1976), 695-696.

[Sh1] R. A. Shore, A decidable class of two quantifier sentences in the theory of the recursively enumerable degrees, vol. 24 Notices Amer. Math. Soc. (1977), A-436. Abstract \# 77T-E48.

[Sh2] __, Nowhere simple sets and the lattice of recursively enumerable sets, J. Symbolic Logic 43 (1978), 322-330.

[Sh3] _ Determining automorphisms of the recursively enumerable sets, Proc. Amer. Math. Soc. 65 (1977), 318-325.

[Sh4] , a-recursion theory, Handbook of Mathematical Logic, J. Barwise, ed., NorthHolland, Amsterdam, 1978.

[Si] S. G. Simpson, Degrees of unsolvability: A survey of results, Handbook of Mathematical Logic, J. Barwise, ed., North-Holland, Amsterdam, 1978.

[Sm] R. M. Smullyan, Effectively simple sets, Proc. Amer. Math Soc. 15 (1964), 893-894.

[So1] R. I. Soare, The Friedberg-Muchnik Theorem re-examined, Canad. J. Math. 24 (1972), 1070-1078.

[S02] _ Automorphisms of the lattice of recursively enumerable sets, Bull. Amer. Math. Soc. 80 (1974), 53-58.

[S03] _ Automorphisms of the lattice of recursively enumerable sets. Part I: Maximal sets, Ann. of Math. 100 (1974), 80-120. MR 50 \# 10058.

[So4] _., The infinite injury priority method, J. Symbolic Logic 41 (1976), 513-530.

[S05] _ Recursively enumerable sets, Omega Series, Springer-Verlag, Berlin and New York, (to appear).

[So6] __ Automorphisms of the lattice of recursively enumerable sets. Part II: Low sets (to appear).

[So7] __ Post's program and complete recursively enumerable sets (to appear).

[S08] _ Computational complexity, speedable and levelable sets, J. Symbolic Logic 42 (1977), 545-563.

[Sp1] C. Spector, On degrees of recursive unsolvability, Ann. of Math. (2) 64 (1956), 581-592. MR 18 \#1118.

[Sp2] __ Inductively defined sets of natural numbers, Warsaw Symposium on Infinitistic Methods, New York, 1961, pp. 98-102.

[St] M. Stob, Doctoral Dissertation, Univ. of Chicago, Chicago, Ill., 1979.

[Te1] S. Tennenbaum, Degree of unsolvability and the rate of growth of functions, Notices Amer. Math. Soc. 8 (1961), 608.

[Te2] _ Degree of unsolvability and the rate of growth of functions, Proc. Sympos. Math. Theory of Automata, Microwave Res. Inst. Sympos. Ser. vol. 12, Polytechnic Press, Brooklyn, N. Y., 1962.

[Th] S. K. Thomason, Sublattices of the recursively enumerable degrees, Z. Math. Logik Grundlagen Math. 17 (1971), 273-280. MR 45 \#8523. 
[Ts] R. E. Tulloss, Some complexities of simplicity: concerning grades of simplicity of recursively enumerable sets, Doctoral Dissertation, Univ. of Calif., Berkeley, Calif., 1971.

[Tu] A. M. Turing, On computable numbers, with an application to the Entscheidungsproblem, Proc. London Math. Soc. 42 (1936), 230-265; ibid. 43 (1936), 544-546.

[U] V. A. Uspenskii, Some notes on recursively enumerable sets, $Z$. Math. Logik Grundlagen Math. 3 (1957), 157-170; English transl., Amer. Math. Soc. Transl. (2) 23 (1963), 89-101.

[Y1] C. E. M. Yates, Recursively enumerable sets and retracing functions, Z. Math. Logik Grundlagen Math. 8 (1962), 331-345.

[Y2] Three theorems on the degree of recursively enumerable sets, Duke Math. J. 32 (1965), 461-468. MR 31 \#4721.

[Y3] __, A minimal pair of r.e. degrees, J. Symbolic Logic 31 (1966), 159-168. MR 34 \#5677.

[Y4] _, On the degrees of index sets, Trans. Amer. Math. Soc. 121 (1966), 309-328.

[Y5] , Recursively enumerable degrees and the degrees less than $0^{\prime}$, Sets, Models and Recursion Theory, North-Holland, Amsterdam, 1967, pp. 264-271.

[Y6] _ On the degrees of index sets. II. Trans. Amer. Math. Soc. 135 (1969), 249-266.

[Y7] _ Initial segments of the degrees of unsolvability, Part I, Mathematical Logic and the Foundations of Set Theory (Jerusalem), North-Holland, Amsterdam, 1970, pp. 63-83.

[Y8] __ Initial segments and implications for the structure of degrees, Conference in Mathematical Logic-London 1970, Lecture Notes in Math., no. 255, Springer-Verlag, Berlin and New York, 1972, pp. 305-335.

[Y9] __ Prioric games and minimal degrees below 0, Fund. Math. 82 (1974), 217-237.

[Y10] _ Banach-Mazur games, comeager sets, and degrees of unsolvability, Math. Proc. Cambridge Philos. Soc. (to appear).

Department of Mathematics, University of Chicago, Chicago, Illinois 60637 